\title{
Rhodium-Catalyzed Reaction of Thiols with Polychloroalkanes in the Presence of Triethylamine
}

\author{
Ken Tanaka* and Kaori Ajiki \\ Department of Applied Chemistry, Graduate School of Engineering, Tokyo University of \\ Agriculture and Technology, Koganei, Tokyo 184-8588, Japan
}

\section{General}

Anhydrous $\mathrm{CH}_{2} \mathrm{Cl}_{2}$ (No. 27,099-7) and $\left(\mathrm{CH}_{2} \mathrm{Cl}\right)_{2}$ (No. 28,450-5) were obtained from Aldrich and used as received. TOYOZYME LIP was obtained from TOYOBO ENZYMES. All other reagents were obtained from commercial sources and used as received (without degassing and dehydrating). All reactions were carried out under an atmosphere of argon in oven-dried glassware with magnetic stirring.

\section{Reaction of Thiols with Polychloroalkanes (Table 2 and Scheme 4)}

General Procedure 1 (Table 2, entry 1): Under an Ar atmosphere, $\mathrm{CH}_{2} \mathrm{Cl}_{2}(0.5 \mathrm{~mL})$ solution of $\mathrm{RhCl}\left(\mathrm{PPh}_{3}\right)_{3}(23.1 \mathrm{mg}, 0.0250 \mathrm{mmol})$ was added to $\mathrm{CH}_{2} \mathrm{Cl}_{2}(0.5 \mathrm{~mL})$ solution of octanethiol (73.1 $\mathrm{mg}, 0.500 \mathrm{mmol})$ and $\mathrm{Et}_{3} \mathrm{~N}(0.5 \mathrm{~mL})$ by using $\mathrm{CH}_{2} \mathrm{Cl}_{2}(1.0 \mathrm{~mL})$. The mixture was stirred at $\mathrm{rt}$ for $24 \mathrm{~h}$. The resulting solution was concentrated and purified by preparative TLC (hexane), which furnished 1-octylsulfanylmethylsulfanyloctane $(61.7 \mathrm{mg}, 0.203 \mathrm{mmol}, 81 \%)$ as a colorless oil.

1-Octylsulfanylmethylsulfanyloctane (Table 2 , entry 1). ${ }^{1}$

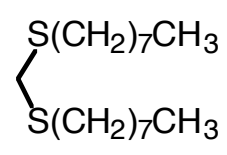

Colorless oil; ${ }^{1} \mathrm{H} \mathrm{NMR}\left(\mathrm{CDCl}_{3}, 300 \mathrm{MHz}\right) \delta 3.65(\mathrm{~s}, 2 \mathrm{H}), 2.62(\mathrm{t}, J=7.2 \mathrm{~Hz}, 4 \mathrm{H}), 1.59$ (quint, $J=$ $7.2 \mathrm{~Hz}, 4 \mathrm{H}), 1.16-1.45(\mathrm{~m}, 20 \mathrm{H}), 0.88(\mathrm{t}, J=6.8 \mathrm{~Hz}, 6 \mathrm{H}) ;{ }^{13} \mathrm{C} \mathrm{NMR}\left(\mathrm{CDCl}_{3}, 75 \mathrm{MHz}\right) \delta 35.5$, $31.8,30.9,29.2,29.1,28.9,22.6,14.0$.

1-Dodecylsulfanylmethylsulfanyldodecane (Table 2, entry 2). ${ }^{2}$ 


$$
\sum_{\mathrm{S}\left(\mathrm{CH}_{2}\right)_{11} \mathrm{CH}_{3}}^{\mathrm{S}\left(\mathrm{CH}_{2}\right)_{11} \mathrm{CH}_{3}}
$$

Colorless solid; Mp 34-35 ${ }^{\circ} \mathrm{C} ;{ }^{1} \mathrm{H}$ NMR $\left(\mathrm{CDCl}_{3}, 300 \mathrm{MHz}\right) \delta 3.65$ (s, 2H), 2.62 (t, J= 7.2 Hz, $4 \mathrm{H}$ ), 1.59 (quint, $J=7.2 \mathrm{~Hz}, 4 \mathrm{H}), 1.20-1.45(\mathrm{~m}, 36 \mathrm{H}), 0.88(\mathrm{t}, J=6.8 \mathrm{~Hz}, 6 \mathrm{H}) ;{ }^{13} \mathrm{C} \mathrm{NMR}\left(\mathrm{CDCl}_{3}\right.$, $75 \mathrm{MHz}) \delta 35.5,31.9,30.9,29.64,29.62,29.59,29.53,29.3,29.2,29.1,28.9,22.7,14.1$.

\section{1,1'-[Methylenebis(thiomethylene)]bisbenzene (Table 2, entry 3). ${ }^{3}$}

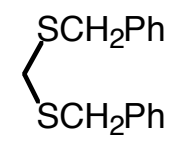

Colorless oil; ${ }^{1} \mathrm{H}$ NMR $\left(\mathrm{CDCl}_{3}, 300 \mathrm{MHz}\right) \delta$ 7.19-7.34 (m, 10H), $3.82(\mathrm{~s}, 4 \mathrm{H}), 3.37(\mathrm{~s}, 2 \mathrm{H}) ;{ }^{13} \mathrm{C}$ NMR $\left(\mathrm{CDCl}_{3}, 75 \mathrm{MHz}\right) \delta 137.7,129.1,128.5,127.0,34.4,33.4$.

\section{1-(11-Hydroxyundecylsulfanylmethylsulfanyl)undecan-1-ol (Table 2, entry 4).}

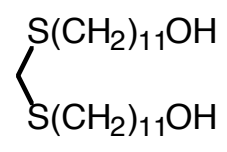

Colorless solid; $\mathrm{Mp} \mathrm{87-88}{ }^{\circ} \mathrm{C}$; IR (neat) 3200, $1600 \mathrm{~cm}^{-1} ;{ }^{1} \mathrm{H}$ NMR ( $\mathrm{CDCl}_{3}, 300 \mathrm{MHz}$, $\delta$ 3.59$3.68(\mathrm{~m}, 6 \mathrm{H}), 2.62(\mathrm{t}, J=7.4 \mathrm{~Hz}, 4 \mathrm{H}), 1.51-1.64(\mathrm{~m}, 8 \mathrm{H}), 1.20-1.44(\mathrm{~m}, 28 \mathrm{H}) ;{ }^{13} \mathrm{C} \mathrm{NMR}\left(\mathrm{CDCl}_{3}\right.$, $75 \mathrm{MHz}) \delta 63.1,35.4,32.8,30.9,29.54,29.47,29.38,29.2,29.1,28.9,25.7$. HRMS (EI, ditrifluoroacetate): calcd for $\mathrm{C}_{27} \mathrm{H}_{46} \mathrm{~S}_{2} \mathrm{O}_{4} \mathrm{~F}_{6}[\mathrm{M}]^{+}$, 612.2743; found, 612.2692.

2-t-Butoxycarbonylamino-3-(2-t-butoxycarbonylamino-2-methoxycarbonylethylsulfanylmethylsulfanyl)propionic acid methylester (Table 2, entry 5).

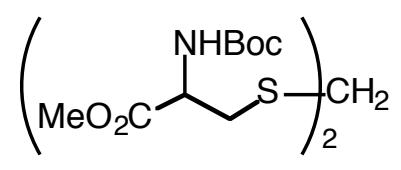

Colorless oil; IR (neat) 3320, 2930, 1690, 1495, 1350, 1150, $1030 \mathrm{~cm}^{-1} ;{ }^{1} \mathrm{H} \mathrm{NMR}\left(\mathrm{CDCl}_{3}, 300\right.$ $\mathrm{MHz}) \delta 5.33-5.44(\mathrm{~m}, 2 \mathrm{H}), 4.49-4.61(\mathrm{~m}, 2 \mathrm{H}), 3.77$ (s, 6H), 3.68 (s, 2H), 3.14 (dd, $J=14.1,4.8$ 
$\mathrm{Hz}, 2 \mathrm{H}), 3.00(\mathrm{dd}, J=14.1,5.9 \mathrm{~Hz}, 2 \mathrm{H}), 1.45(\mathrm{~s}, 18 \mathrm{H}) ;{ }^{13} \mathrm{C} \mathrm{NMR}\left(\mathrm{CDCl}_{3}, 75 \mathrm{MHz}\right) \delta 171.4$, 155.0, 80.2, 53.0, 52.5, 36.4, 33.0, 28.2. Anal. Calcd for $\mathrm{C}_{19} \mathrm{H}_{34} \mathrm{~N}_{2} \mathrm{O}_{8} \mathrm{~S}_{2}: \mathrm{C}, 47.28 ; \mathrm{H}, 7.10 ; \mathrm{N}, 5.80$. Found: C, 47.48; H, 7.45; N, 5.46.

1-p-Tolylsulfanylmethylsulfanyl-p-toluene (Table 2 , entry 6$).^{4}$

$$
\left\langle\begin{array}{l}
S(p-T o l) \\
S(p-T o l)
\end{array}\right.
$$

Colorless oil; ${ }^{1} \mathrm{H}$ NMR $\left(\mathrm{CDCl}_{3}, 300 \mathrm{MHz}\right) \delta$ 7.29-7.36 (m, 4H), 7.12 (d, J= 7.8 Hz, 4H), 4.26 (s, 2H), $2.33(\mathrm{~s}, 6 \mathrm{H}) ;{ }^{13} \mathrm{C} \mathrm{NMR}\left(\mathrm{CDCl}_{3}, 75 \mathrm{MHz}\right) \delta 137.4,131.5,131.3,129.7,42.0,21.1$.

1-(2-Octylsulfanylethylsulfanyl)octane (Table2, entry 7). 5

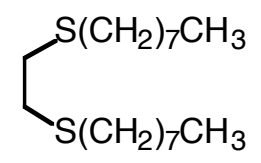

Reaction temperature: $80^{\circ} \mathrm{C}$.

Colorless oil; ${ }^{1} \mathrm{H} \mathrm{NMR}\left(\mathrm{CDCl}_{3}, 300 \mathrm{MHz}\right) \delta 2.71(\mathrm{~s}, 4 \mathrm{H}), 2.54(\mathrm{t}, J=7.5 \mathrm{~Hz}, 4 \mathrm{H}), 1.59$ (quint, $J=$ $7.2 \mathrm{~Hz}, 4 \mathrm{H}), 1.22-1.43(\mathrm{~m}, 20 \mathrm{H}), 0.88(\mathrm{t}, J=6.8 \mathrm{~Hz}, 6 \mathrm{H}) ;{ }^{13} \mathrm{C} \mathrm{NMR}\left(\mathrm{CDCl}_{3}, 75 \mathrm{MHz}\right) \delta 32.2$, $32.1,31.8,29.7,29.2,28.9,22.6,14.1$.

\section{1-p-Tolylsulfanylethylsulfanyl-p-toluene (Table2, entry 8). ${ }^{6}$}

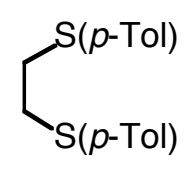

Reaction temperature: $80^{\circ} \mathrm{C}$.

Colorless solid; Mp 74-76 ${ }^{\circ} \mathrm{C} ;{ }^{1} \mathrm{H}$ NMR $\left(\mathrm{CDCl}_{3}, 300 \mathrm{MHz}\right) \delta$ 7.19-7.24 (m, 4H), 7.05-7.12 (m, 4H), $3.01(\mathrm{~s}, 4 \mathrm{H}), 2.32(\mathrm{~s}, 6 \mathrm{H}) ;{ }^{13} \mathrm{C} \mathrm{NMR}\left(\mathrm{CDCl}_{3}, 75 \mathrm{MHz}\right) \delta 136.7,131.3,130.7,129.8,34.0$, 21.0.

Thioformic acid $S$-dodecylester (Table2, entry 9). 


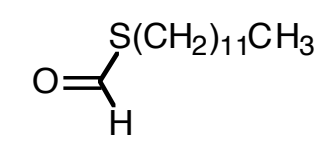

Procedure 1 was followed using $\mathrm{RhCl}\left(\mathrm{PPh}_{3}\right)_{3}(92.5 \mathrm{mg}, 0.100 \mathrm{mmol})$, dodecanethiol (101.2 mg, $0.500 \mathrm{mmol}), \mathrm{Et}_{3} \mathrm{~N}(0.55 \mathrm{~mL})$, and $\mathrm{CHCl}_{3}(10 \mathrm{~mL})$, which furnished thioformic acid $S$-dodecylester (26.5 mg, $0.115 \mathrm{mmol}, 69 \%$ ).

Colorless oil; IR (neat) 2900, 1670, $1450 \mathrm{~cm}^{-1}$; ${ }^{1} \mathrm{H}$ NMR $\left(\mathrm{CDCl}_{3}, 300 \mathrm{MHz}\right.$, $\delta 10.13$ (s, 1H), 2.98 (t, $J=7.2 \mathrm{~Hz}, 2 \mathrm{H}$ ), 1.61 (quint, $J=7.5 \mathrm{~Hz}, 2 \mathrm{H}), 1.17-1.42(\mathrm{~m}, 18 \mathrm{H}), 0.88(\mathrm{t}, J=6.8 \mathrm{~Hz}, 3 \mathrm{H}) ;{ }^{13} \mathrm{C}$ NMR $\left(\mathrm{CDCl}_{3}, 75 \mathrm{MHz}\right) \delta 187.8,31.9,29.59,29.52,29.45,29.42,29.3,29.0,28.7,26.7,22.7,14.1$. HRMS (EI): calcd for $\mathrm{C}_{12} \mathrm{H}_{25} \mathrm{~S}$ [M-CHO] ${ }^{+}, 201.1677$; found, 201.1659.

\section{1-Deuteriumthioformic acid $S$-dodecylester (Scheme 4).}

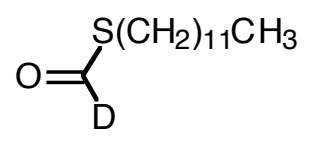

Procedure 1 was followed using $\mathrm{RhCl}\left(\mathrm{PPh}_{3}\right)_{3}(92.5 \mathrm{mg}, 0.100 \mathrm{mmol})$, dodecanethiol (101.2 mg, $0.500 \mathrm{mmol}), \mathrm{Et}_{3} \mathrm{~N}(0.55 \mathrm{~mL})$, and $\mathrm{CDCl}_{3}(10 \mathrm{~mL})$, which furnished 1-deuteriumthioformic acid $S$ dodecylester (23.4 mg, $0.102 \mathrm{mmol}, 61 \%)$.

Colorless oil; IR (neat) 2900, 1670, $1450 \mathrm{~cm}^{-1} ;{ }^{1} \mathrm{H} \mathrm{NMR}\left(\mathrm{CDCl}_{3}, 300 \mathrm{MHz}\right.$, $) 2.98$ (t, J = 7.2 Hz, $2 \mathrm{H}$ ), 1.61 (quint, $J=7.2 \mathrm{~Hz}, 2 \mathrm{H}), 1.17-1.42(\mathrm{~m}, 18 \mathrm{H}), 0.88(\mathrm{t}, J=6.8 \mathrm{~Hz}, 3 \mathrm{H}) ;{ }^{2} \mathrm{H} \mathrm{NMR}\left(\mathrm{CDCl}_{3}\right.$, $61 \mathrm{MHz}) \delta 10.17$ (s, 1D).

Ditiocarbonic acid $S, S$-dihexylester (Table2, entry 10).

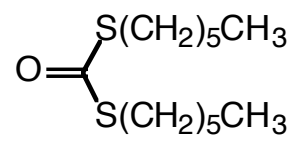

Procedure 1 was followed using $\mathrm{RhCl}\left(\mathrm{PPh}_{3}\right)_{3}(92.5 \mathrm{mg}, 0.100 \mathrm{mmol})$, hexanethiol $(59.1 \mathrm{mg}, 0.500$ $\mathrm{mmol}), \mathrm{Et}_{3} \mathrm{~N}(0.55 \mathrm{~mL})$, and $\mathrm{CCl}_{4}(10 \mathrm{~mL})$, which furnished ditiocarbonic acid $S, S$-dioctylester (20.1 mg, $0.0766 \mathrm{mmol}, 61 \%)$.

Colorless oil; IR (neat) 2940, 1640, 1450, $870 \mathrm{~cm}^{-1} ;{ }^{1} \mathrm{H} \mathrm{NMR}\left(\mathrm{CDCl}_{3}, 300 \mathrm{MHz},\right) \delta 2.98(\mathrm{t}, J=7.2$ $\mathrm{Hz}, 4 \mathrm{H}), 1.61$ (quint, $J=7.2 \mathrm{~Hz}, 4 \mathrm{H}), 1.20-1.44(\mathrm{~m}, 12 \mathrm{H}), 0.88(\mathrm{t}, J=6.8 \mathrm{~Hz}, 6 \mathrm{H}) ;{ }^{13} \mathrm{C} \mathrm{NMR}$ $\left(\mathrm{CDCl}_{3}, 75 \mathrm{MHz}\right) \delta 189.8,31.2,30.6,29.7,28.4,22.5,14.0$. HRMS (EI): calcd for $\mathrm{C}_{13} \mathrm{H}_{26} \mathrm{OS}_{2}$ 
$[\mathrm{M}]^{+}, 262.1425$; found, 262.1397.

\section{Reaction of Thiols with Alkyl Halides (Schemes 2, 3, and 5)}

General Procedure 2 (Scheme 2): Under Ar atmosphere, $\mathrm{Et}_{3} \mathrm{~N}$ (0.25 mL) solution of $\mathrm{RhCl}\left(\mathrm{PPh}_{3}\right)_{3}(11.6 \mathrm{mg}, 0.0125 \mathrm{mmol})$ was added to $\mathrm{Et}_{3} \mathrm{~N}(0.25 \mathrm{~mL})$ solution of benzylchloride (31.6 mg, $0.250 \mathrm{mmol})$ and octanethiol $(36.6 \mathrm{mg}, 0.250 \mathrm{mmol})$ by using $\mathrm{Et}_{3} \mathrm{~N}(0.50 \mathrm{~mL})$. The mixture was stirred for at $80{ }^{\circ} \mathrm{C}$ for $24 \mathrm{~h}$. The resulting solution was concentrated and purified by preparative TLC (hexane), which furnished 1-octylsulfanylmethylbenzene (43.7 mg, $0.185 \mathrm{mmol}$, $74 \%)$ as a colorless oil.

\section{1-Octylsulfanylmethylbenzene (Scheme 2). ${ }^{7}$}

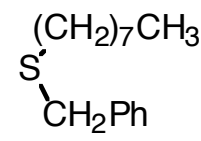

Colorless oil; ${ }^{1} \mathrm{H}$ NMR $\left(\mathrm{CDCl}_{3}, 300 \mathrm{MHz}\right) \delta$ 7.18-7.35 (m, 5H), 3.69 (s, 2H), 2.40 (t, J = 7.2 Hz, $2 \mathrm{H}), 1.54$ (quint, $J=7.2 \mathrm{~Hz}, 2 \mathrm{H}), 1.15-1.40(\mathrm{~m}, 10 \mathrm{H}), 0.88(\mathrm{t}, J=6.8 \mathrm{~Hz}, 3 \mathrm{H}) ;{ }^{13} \mathrm{C} \mathrm{NMR}\left(\mathrm{CDCl}_{3}\right.$, $75 \mathrm{MHz}) \delta 138.6,128.8,128.4,126.8,36.2,31.8,31.3,29.2,29.14,29.12,28.8,22.6,14.1$.

\section{1-Dodecylsulfanylmethylbenzene (Scheme 3). ${ }^{8}$}

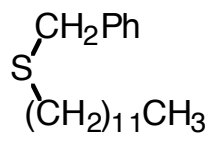

$\mathrm{RhCl}\left(\mathrm{PPh}_{3}\right)_{3}(2.3 \mathrm{mg}, 0.0025 \mathrm{mmol})$ was used. Reaction temperature: $50{ }^{\circ} \mathrm{C}$. Rection time: $30 \mathrm{~h}$. Colorless oil; ${ }^{1} \mathrm{H}$ NMR $\left(\mathrm{CDCl}_{3}, 300 \mathrm{MHz}\right) \delta$ 7.18-7.36 (m, 5H), $3.70(\mathrm{~s}, 2 \mathrm{H}), 2.40$ (t, J = 7.5 Hz, $2 \mathrm{H}), 1.49-1.60(\mathrm{~m}, 2 \mathrm{H}), 1.18-1.41(\mathrm{~m}, 18 \mathrm{H}), 0.88(\mathrm{t}, J=6.8 \mathrm{~Hz}, 3 \mathrm{H}) ;{ }^{13} \mathrm{C} \mathrm{NMR}\left(\mathrm{CDCl}_{3}, 75 \mathrm{MHz}\right)$ $\delta 138.7,128.8,128.4,126.8,36.3,31.9,31.4,29.63,29.61,29.57,29.48,29.3,29.22$, 29.20, 28.9, $22,7,14.1$.

\section{(S)-(-)-1-Phenylethylbenzylsulfide (78\% ee, Scheme 5). ${ }^{9}$}




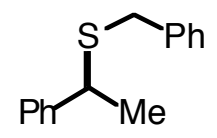

Reaction time: $48 \mathrm{~h}$.

Colorless oil; $[\alpha]^{20}{ }_{\mathrm{D}}-196.9^{\circ}\left(c 0.344, \mathrm{EtOH}, 78 \%\right.$ ee) $\left[\mathrm{lit}^{9}{ }^{9}[\alpha]_{\mathrm{D}}^{20}-83.1^{\circ}(\mathrm{EtOH})\right] ;{ }^{1} \mathrm{H} \mathrm{NMR}$ $\left(\mathrm{CDCl}_{3}, 300 \mathrm{MHz}\right.$, ) 7.18-7.37 (m, 10H), 3.80 (q, $\left.J=7.2 \mathrm{~Hz}, 1 \mathrm{H}\right), 3.54(\mathrm{~d}, J=13.5 \mathrm{~Hz}, 1 \mathrm{H}), 3.43$ $(\mathrm{d}, J=13.5 \mathrm{~Hz}, 1 \mathrm{H}), 1.52(\mathrm{~d}, J=7.2 \mathrm{~Hz}, 3 \mathrm{H}) ;{ }^{13} \mathrm{C} \mathrm{NMR}\left(\mathrm{CDCl}_{3}, 75 \mathrm{MHz}\right) \delta 143.8,138.4,128.8$, $128.5,128.4,127.4,127.0,126.8,43.5,35.7,22.5$. Chiraldex B-DM column, retension time $=37.5$ $\min (R$ isomer $)$ and $38.2 \mathrm{~min}(S$ isomer $)\left(160^{\circ} \mathrm{C}\right.$; isothermal $)$.

\section{Synthsis of (R)-(1-Chloroethyl)benzene (78\% ee, Scheme 5).}

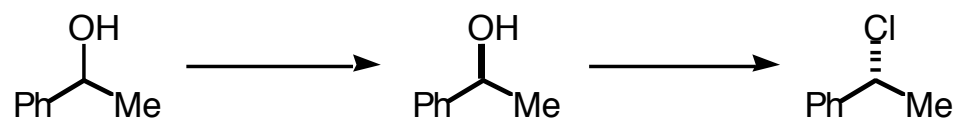

A mixture of methylbenzyl alcohol (1.0 g, $8.2 \mathrm{mmol})$, TOYOZYME LIP (75 mg), vinyl laurate (1.1 $\mathrm{mL})$, and $i-\mathrm{Pr}_{2} \mathrm{O}(10 \mathrm{~mL})$ was stirred at $\mathrm{rt}$ for 2 days. The reaction mixture was filtered and concentrated. The residue was purified by silica gel column chromatography (hexane:EtOAc = 20:1), which furnished $(S)$-methylbenzyl alcohol ( $0.43 \mathrm{~g}, 3.5 \mathrm{mmol}, 43 \%,>99 \%$ ee) as colorless oil. ${ }^{1} \mathrm{H}$ NMR $\left(\mathrm{CDCl}_{3}, 300 \mathrm{MHz}\right) \delta$ 7.23-7.41 (m, 5H), $4.91(\mathrm{dq}, J=6.6,3.5 \mathrm{~Hz}, 1 \mathrm{H}), 1.85-1.93$ (m, $1 \mathrm{H}), 1.50(\mathrm{~d}, J=6.6,3 \mathrm{H})$. Chiraldex G-TA column, retension time $=11.8 \mathrm{~min}(R$ isomer $)$ and 12.3 $\min (S$ isomer $)\left(100{ }^{\circ} \mathrm{C}\right.$; isothermal).

To a solution of $(S)$-methylbenzyl alcohol $(0.21 \mathrm{~g}, 1.7 \mathrm{mmol},>99 \% \mathrm{ee})$, pyridine $(0.16 \mathrm{~mL}, 2.0$ $\mathrm{mmol}), \mathrm{DMF}(0.5 \mathrm{~mL})$, and pentane $(4.5 \mathrm{~mL})$ was added methanesulfonyl chloride $(0.18 \mathrm{~mL} 1.87$ mmol), keeping the temperature below $0{ }^{\circ} \mathrm{C}$, and the resulting mixture was stirred at $\mathrm{rt}$ for $5 \mathrm{~h}$. The mixture was quenched by the addition of ice water, the organic layer was washed with $1 \mathrm{M}$ aqueos $\mathrm{HCl}, 5 \%$ aqueos $\mathrm{NaHCO}_{3}$. The solvent was removed under reduced pressure to give $(R)$ methylbenzyl chloride $(0.12 \mathrm{~g}, 0.85 \mathrm{mmol}, 50 \%, 78 \%$ ee $)$ as a colorless oil; ${ }^{1} \mathrm{H} \mathrm{NMR}\left(\mathrm{CDCl}_{3}, 300\right.$ $\mathrm{MHz}) \delta 7.26-7.46(\mathrm{~m}, 5 \mathrm{H}), 5.10(\mathrm{q}, J=6.8 \mathrm{~Hz}, 1 \mathrm{H}), 1.86(\mathrm{~d}, J=6.6 \mathrm{~Hz}, 3 \mathrm{H})$. Chiraldex G-TA column, retension time $=7.53 \mathrm{~min}(R$ isomer $)$ and $7.80 \mathrm{~min}(S$ isomer $)\left(100{ }^{\circ} \mathrm{C}\right.$; isothermal $)$.

\section{References}

1) Okamoto, Y.; Ohta, H.; Tsuchihashi, G. Chem. Lett. 1986, 12, 2049. 
2) Petrosyan, V. A.; Niyazymbetov, M. E.; Konyushkin, L. D.; Litvinov, V. P. Synthesis 1990, 9, 841.

3) Kuhn, N.; Schumann, H. J. Org. Chem. 1986, 315, 93.

4) Kakimoto, M.; Seri, T.; Imai, Y. Synthesis 1987, 2, 164.

5) Walsh, J. P.; Fahrner, L.; Bell, R. M. J. Biol. Chem. 1990, 265, 4374.

6) Ternay, A. L. Jr.; Lin, J.; Sutliff, T.; Chu, S. S. C.; Chung, B. J. Org. Chem. 1978, 43, 3024.

7) Takido, T.; Itabashi, K. Synthesis. 1987, 9, 817.

8) Yadav, J. S.; Reddy, B. V. S.; Srinivas, C.; Srihari, P. Synlett 2001, 6, 854.

9) Richard, S. G.; Borys H.; Jerry H.-S. L.; Bogdan M.; Joan T.-C. J. Am. Chem. Soc. 1984, 106, 1779. 

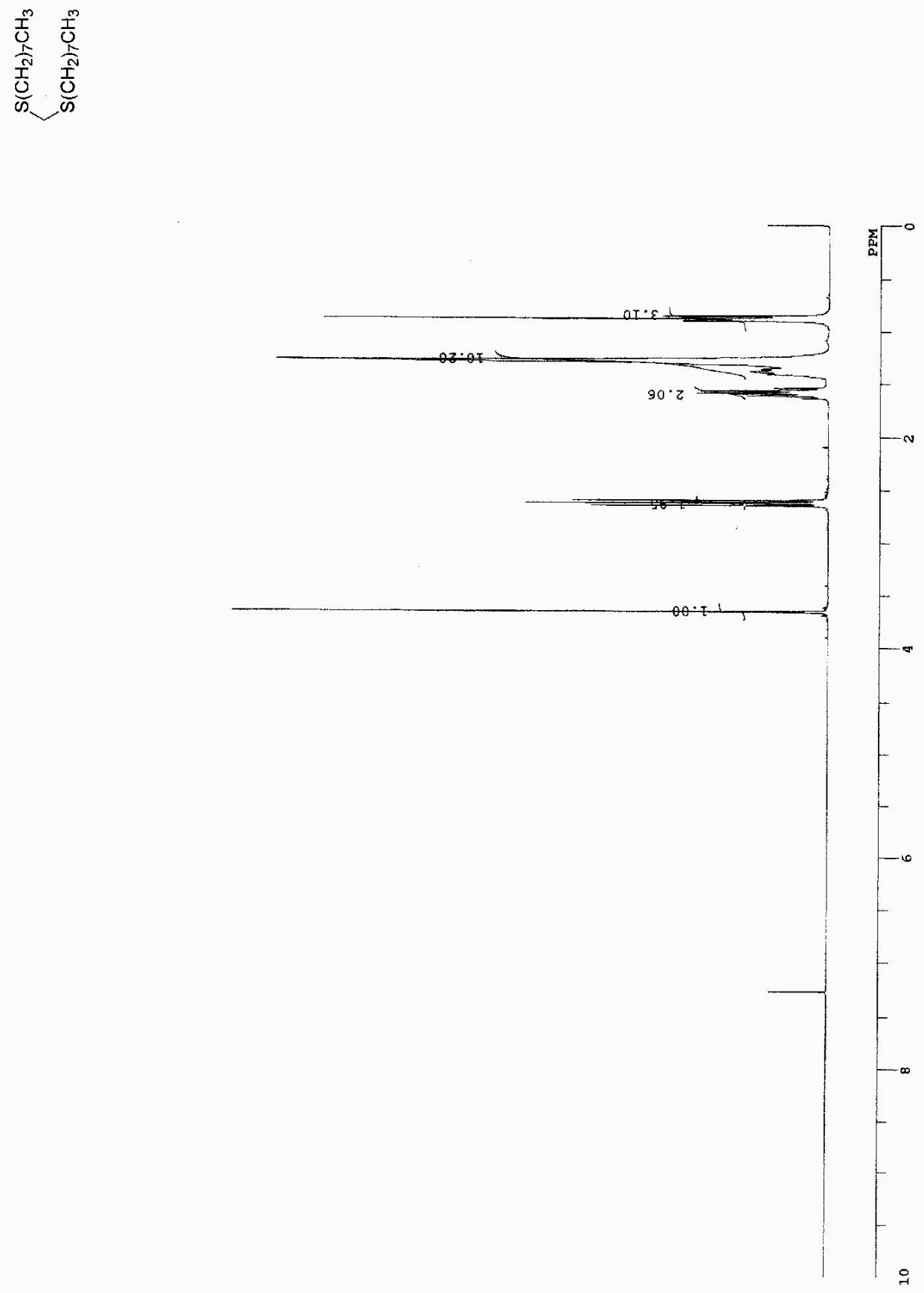

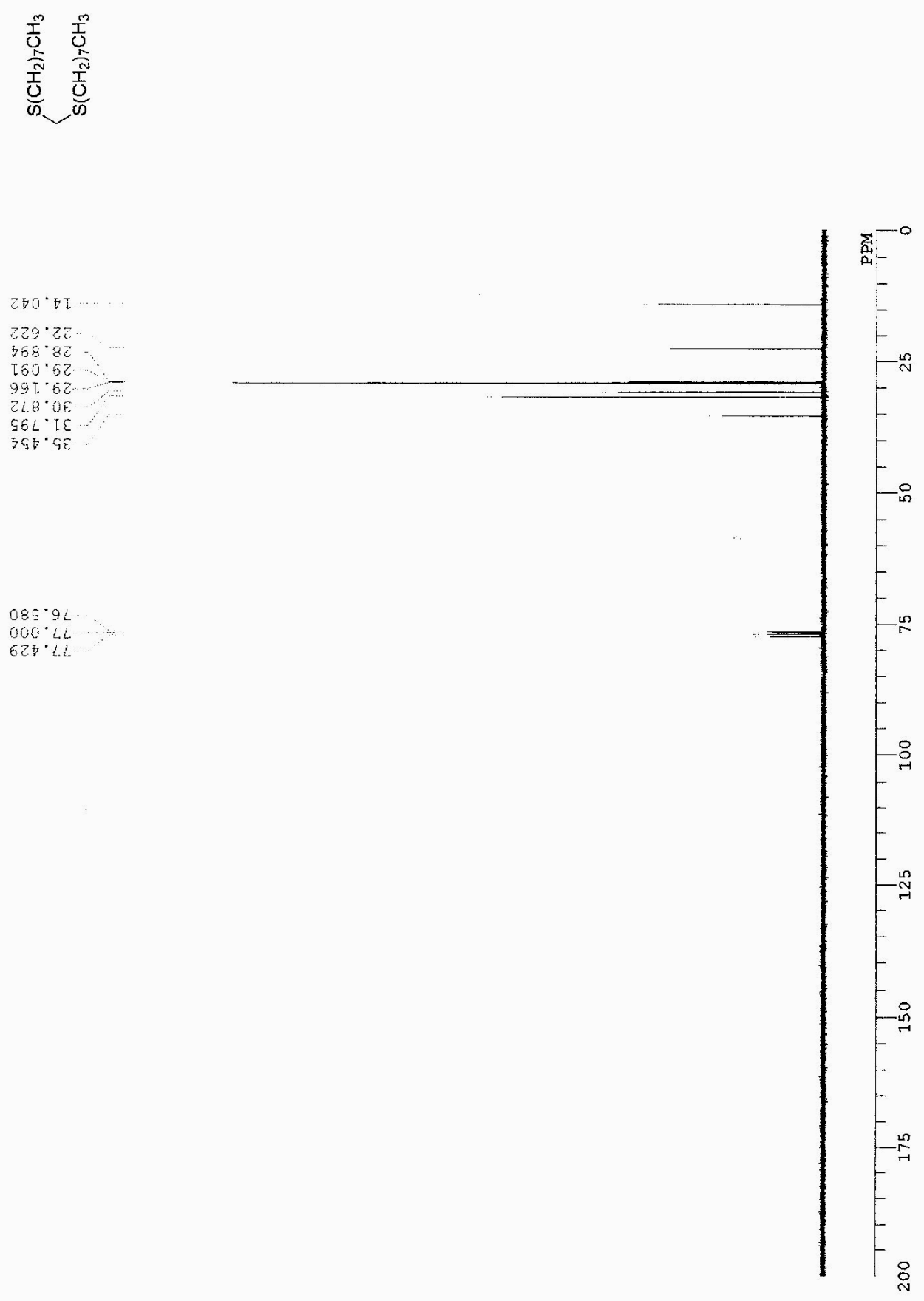


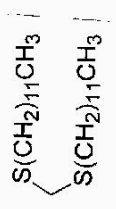

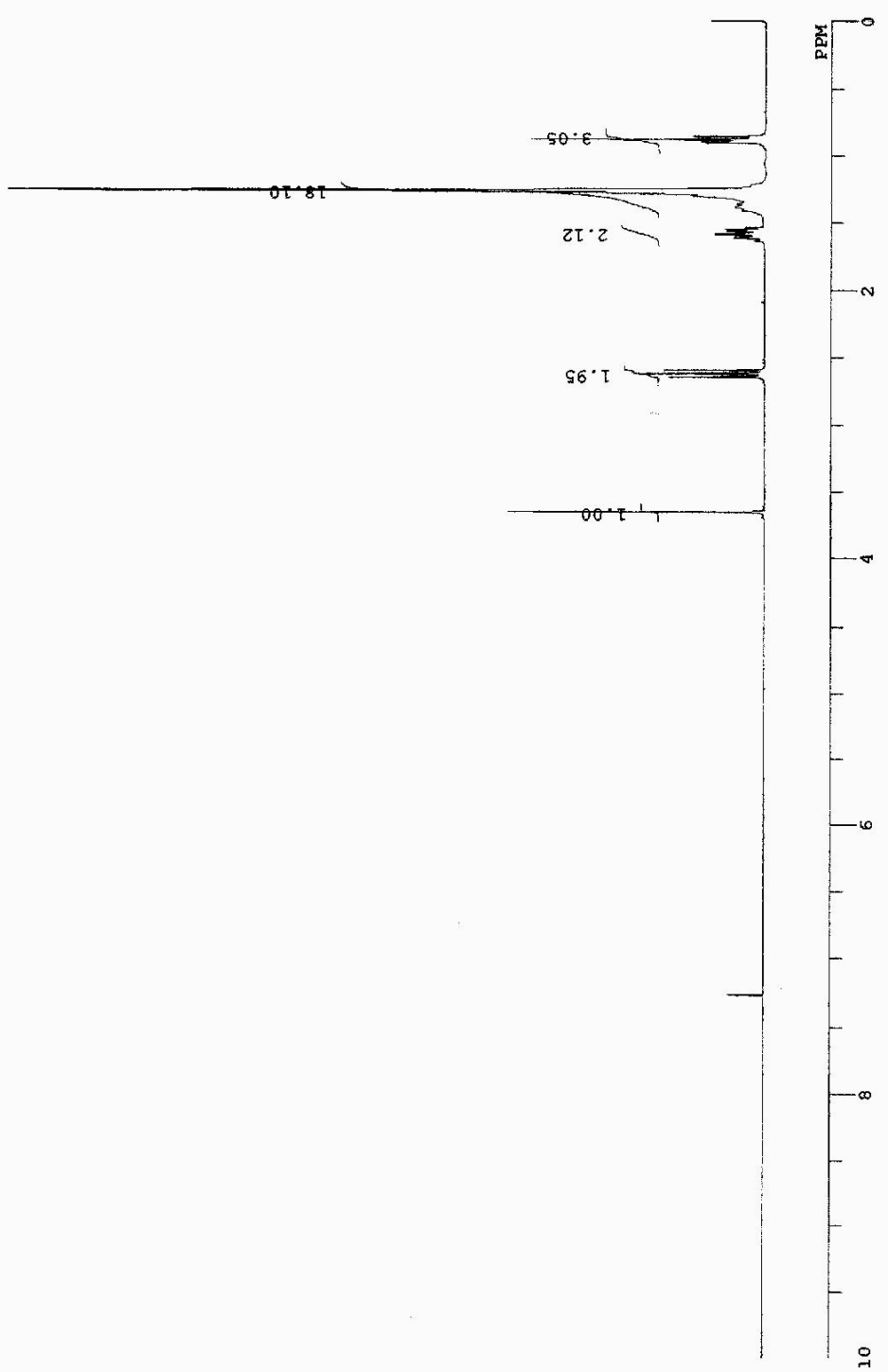



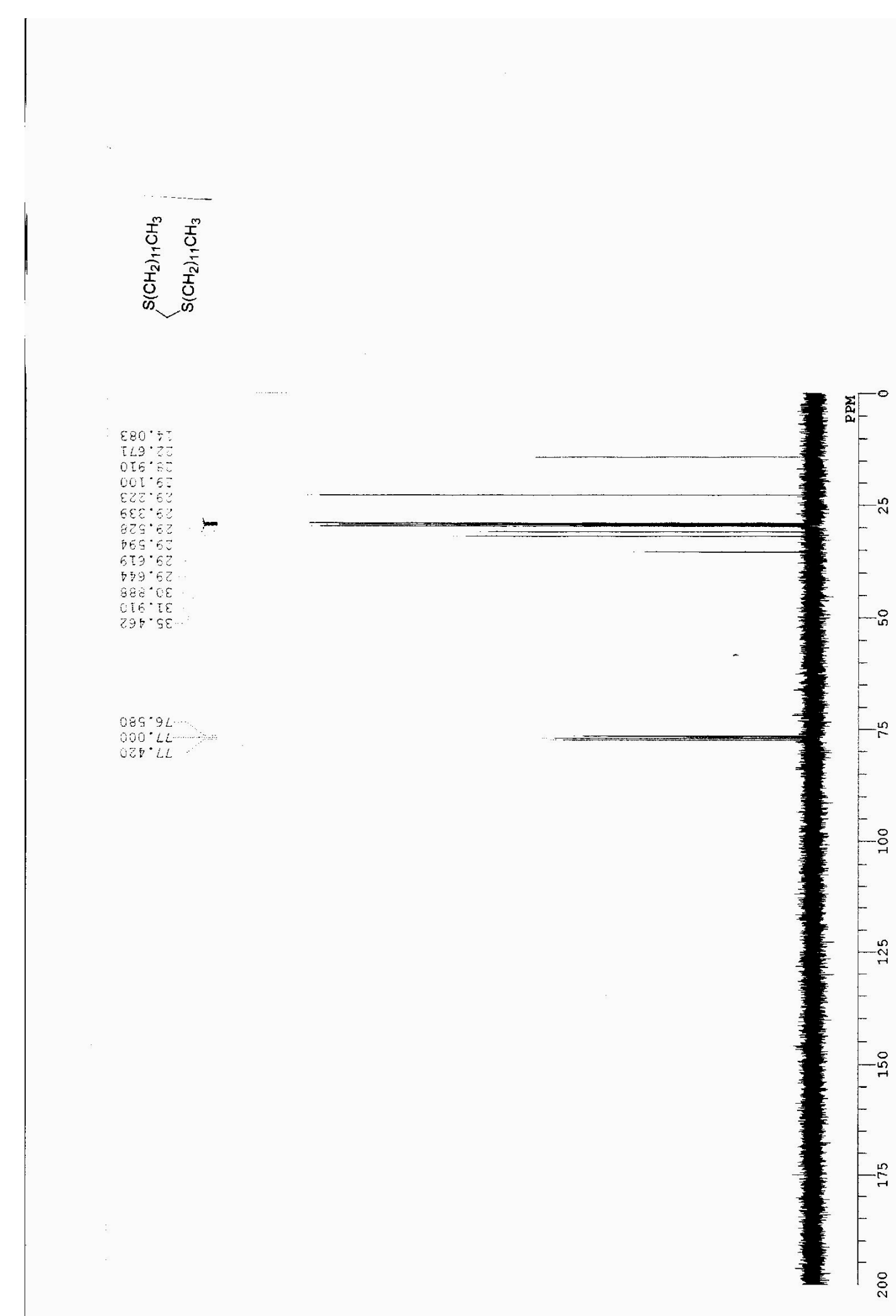


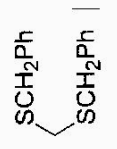

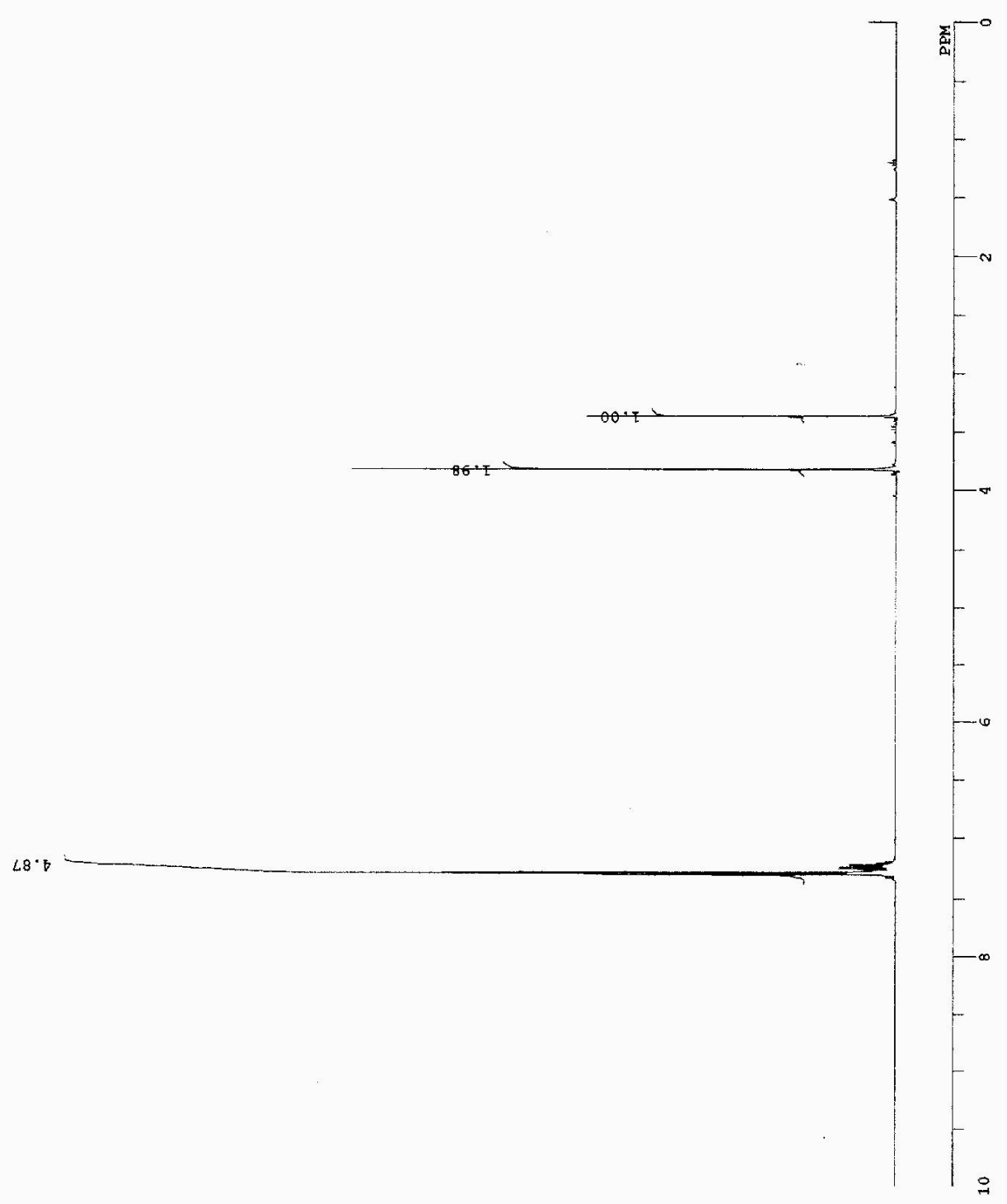




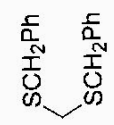

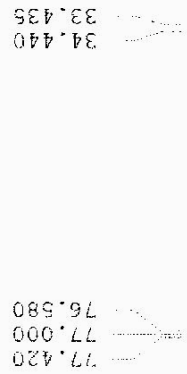

OLO LET.

$209^{\circ} \mathrm{gzt}$

$899^{\circ} \operatorname{L\varepsilon I}$

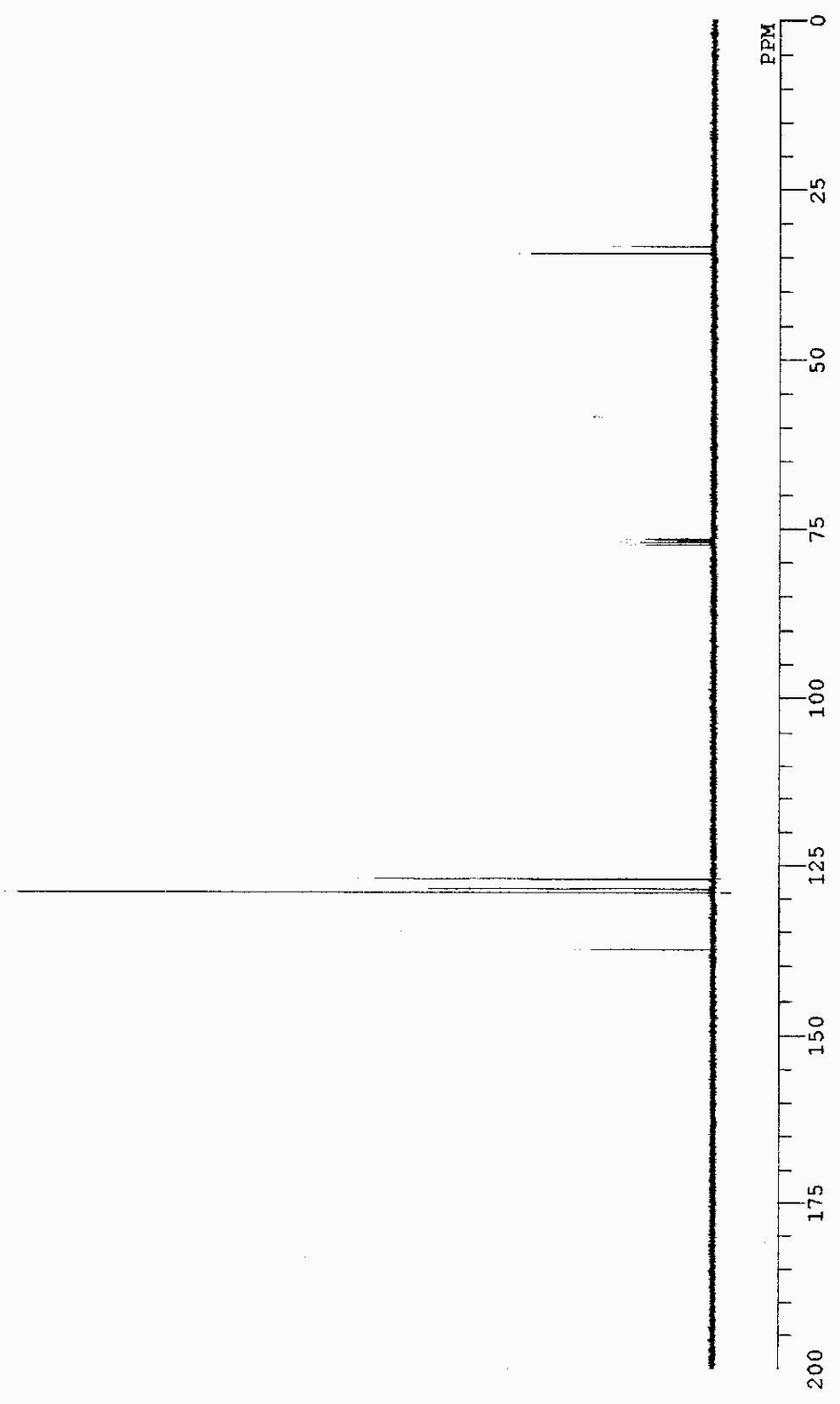




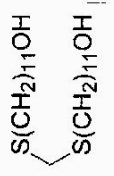

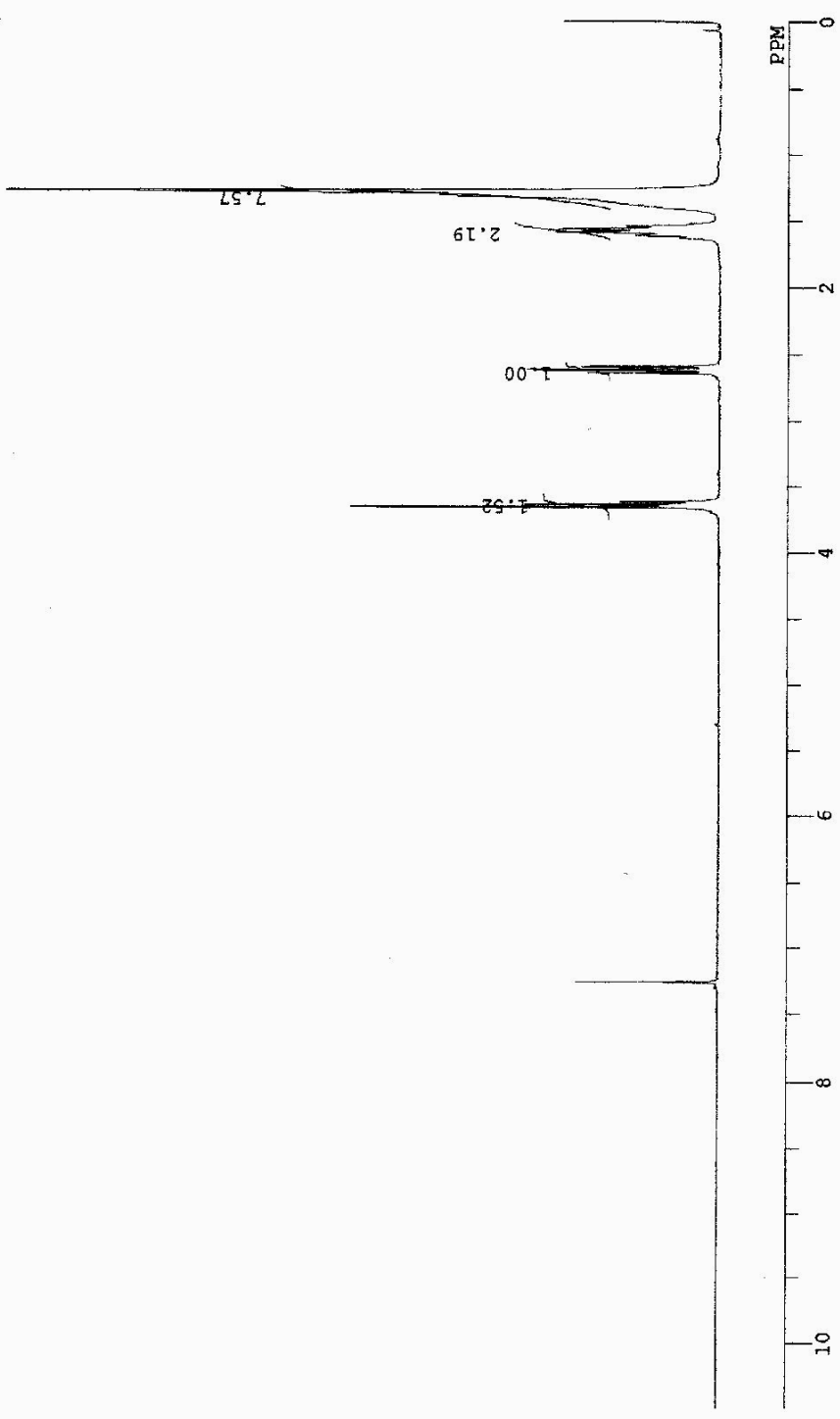




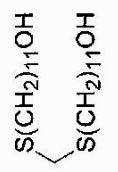

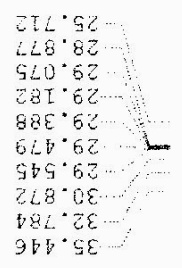

$\varsigma \subsetneq 0^{\circ} \varepsilon 9 \ldots \ldots \ldots$

TLS.9L

$02 t^{\circ} \angle L$

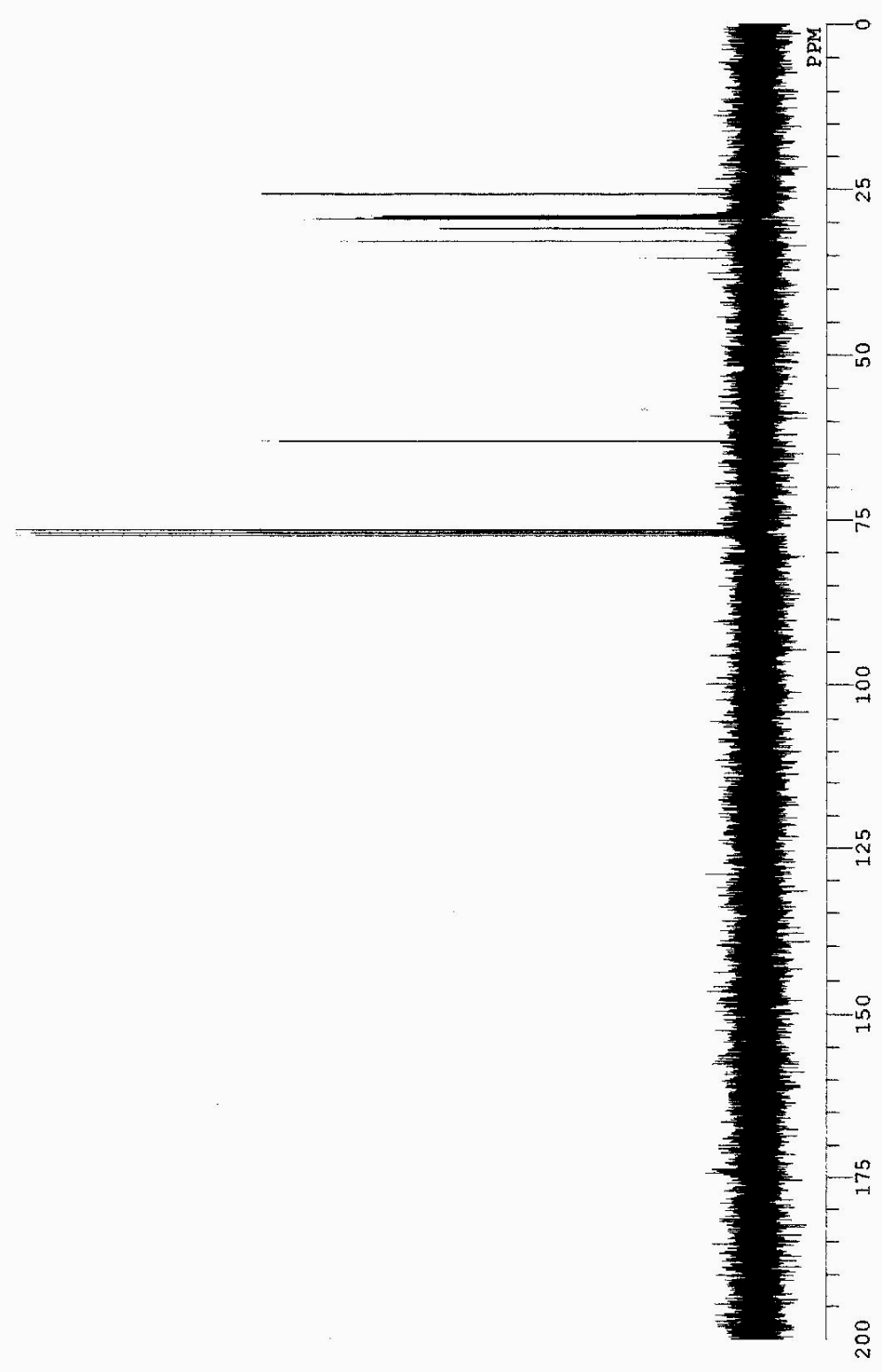



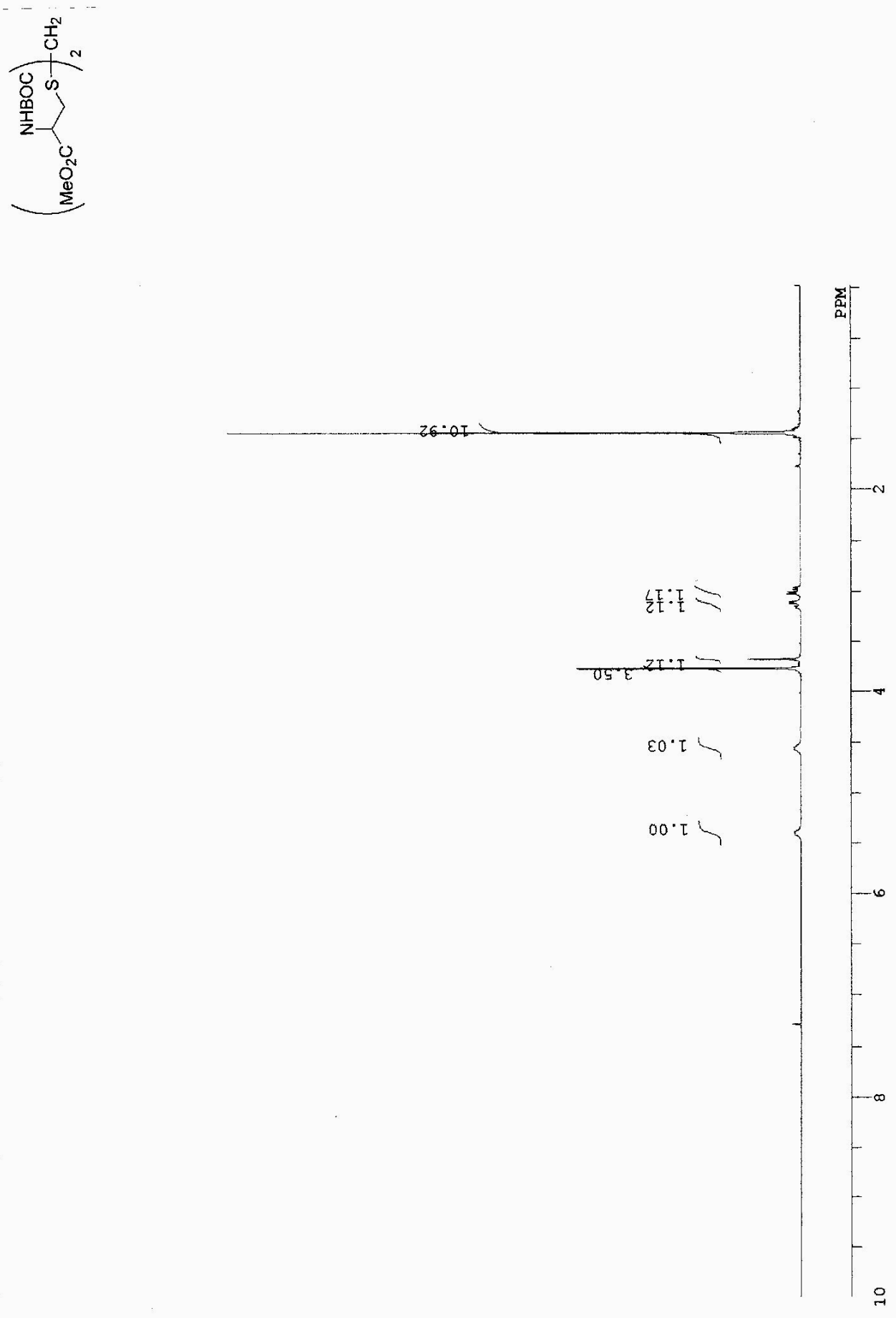

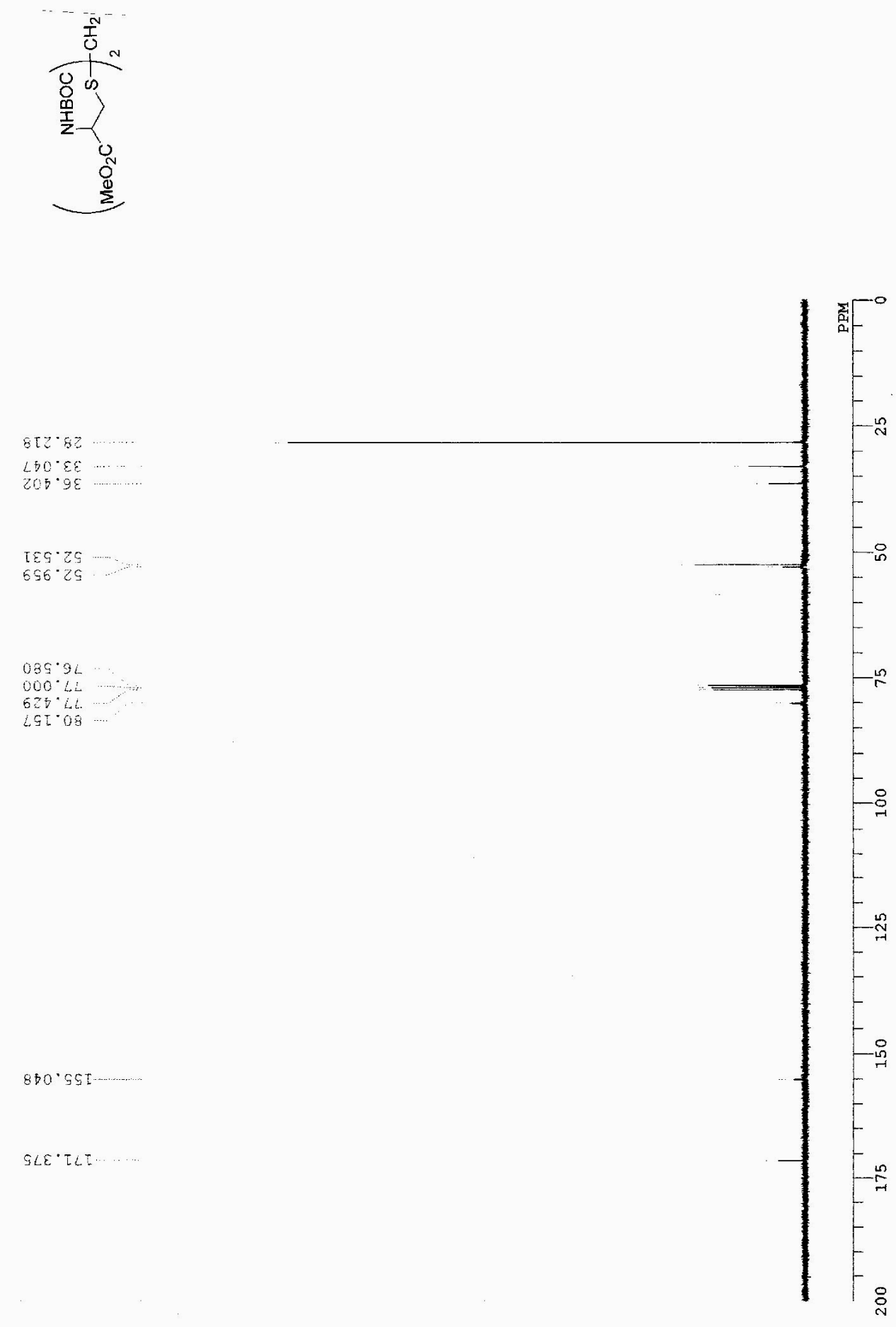
$\overline{\frac{\bar{o}}{\overline{0}}}$

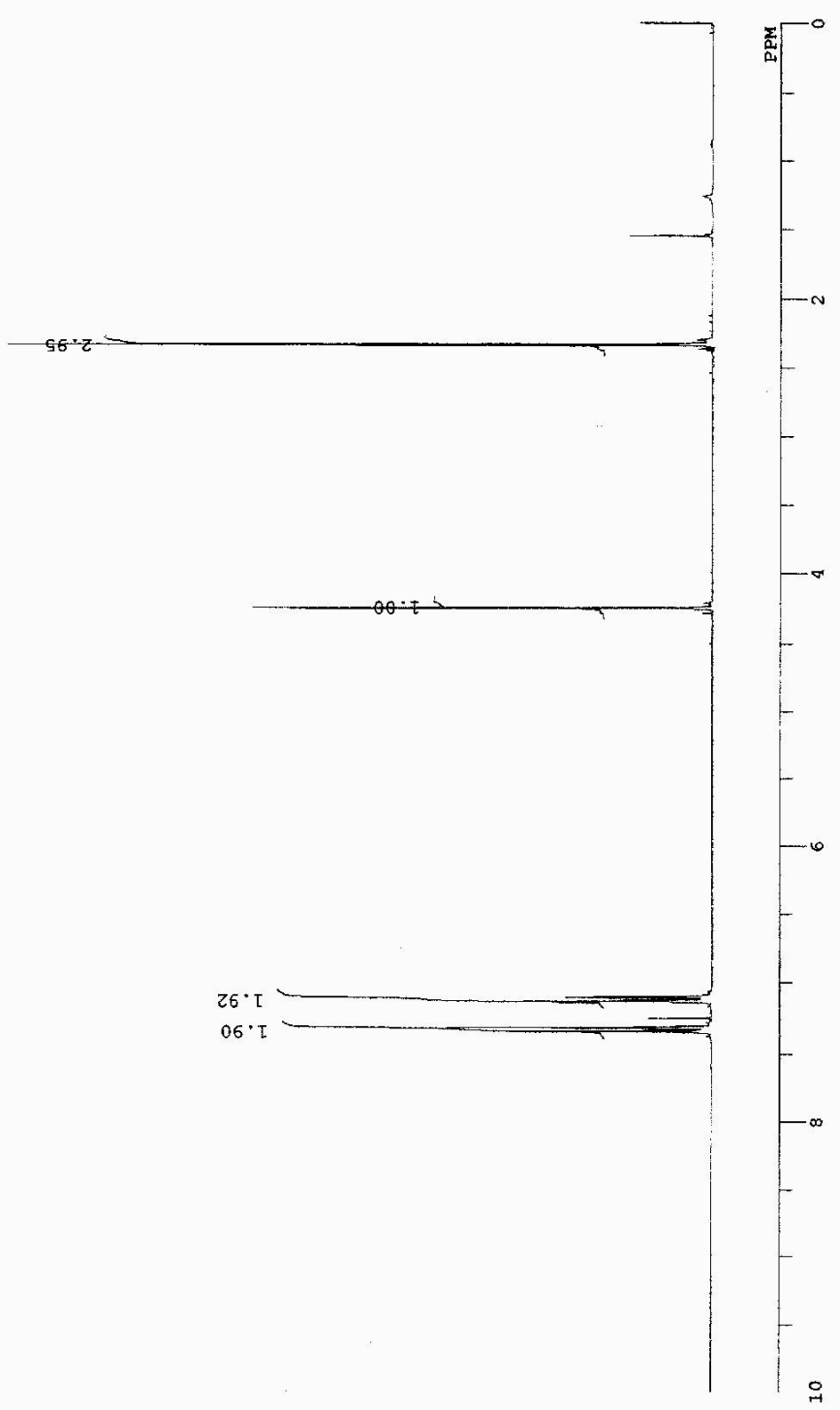




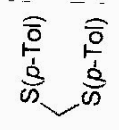

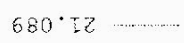

ILG. $9 L$

$000^{\circ} \mathrm{LL}$

$O Z F \cdot L L$

$8 S L .6 Z T \cdot \cdots \cdot$.

โ8D. [E]....

$\varsigma \varepsilon \cdot$ L $\mathrm{T}$

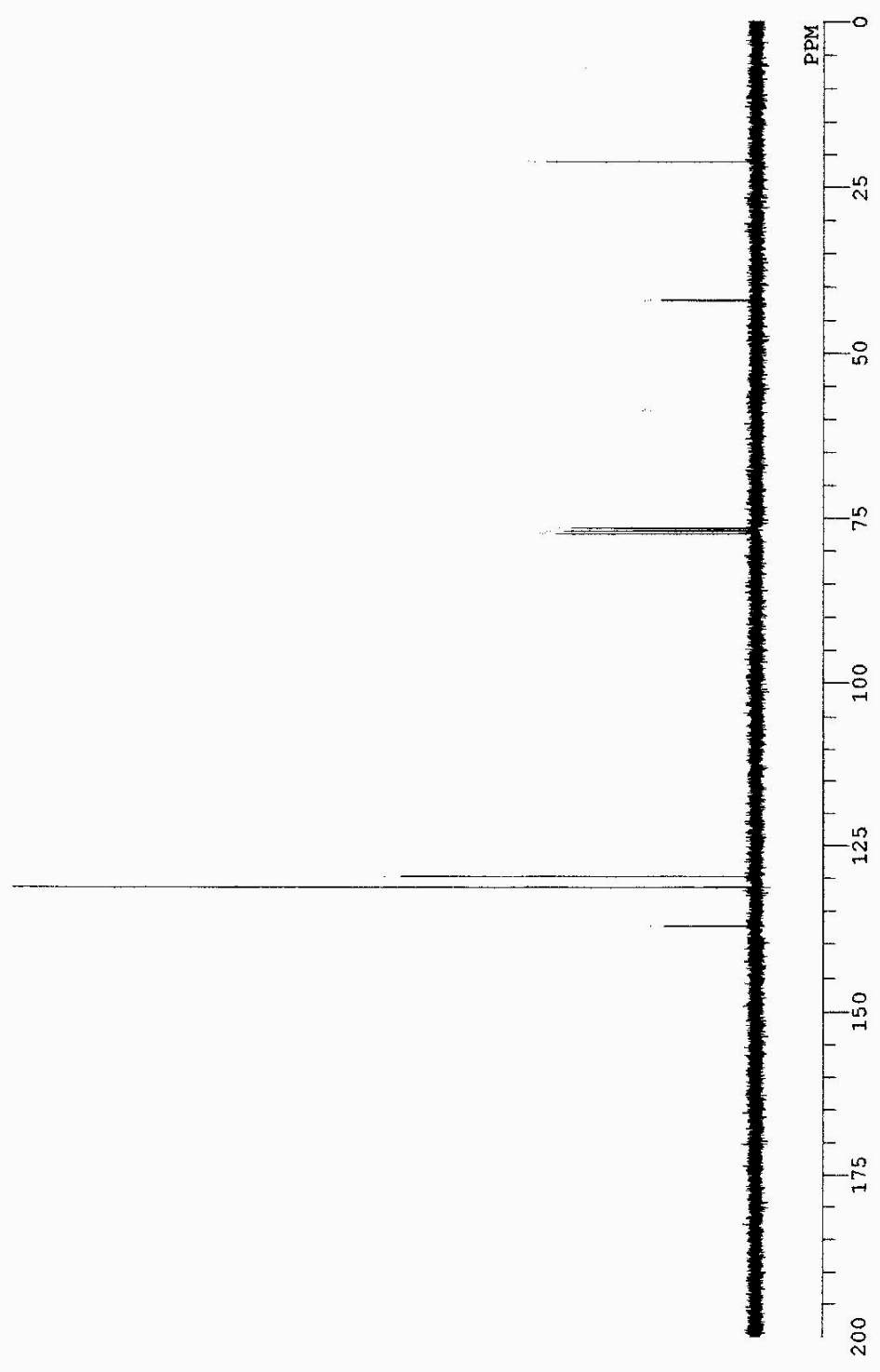



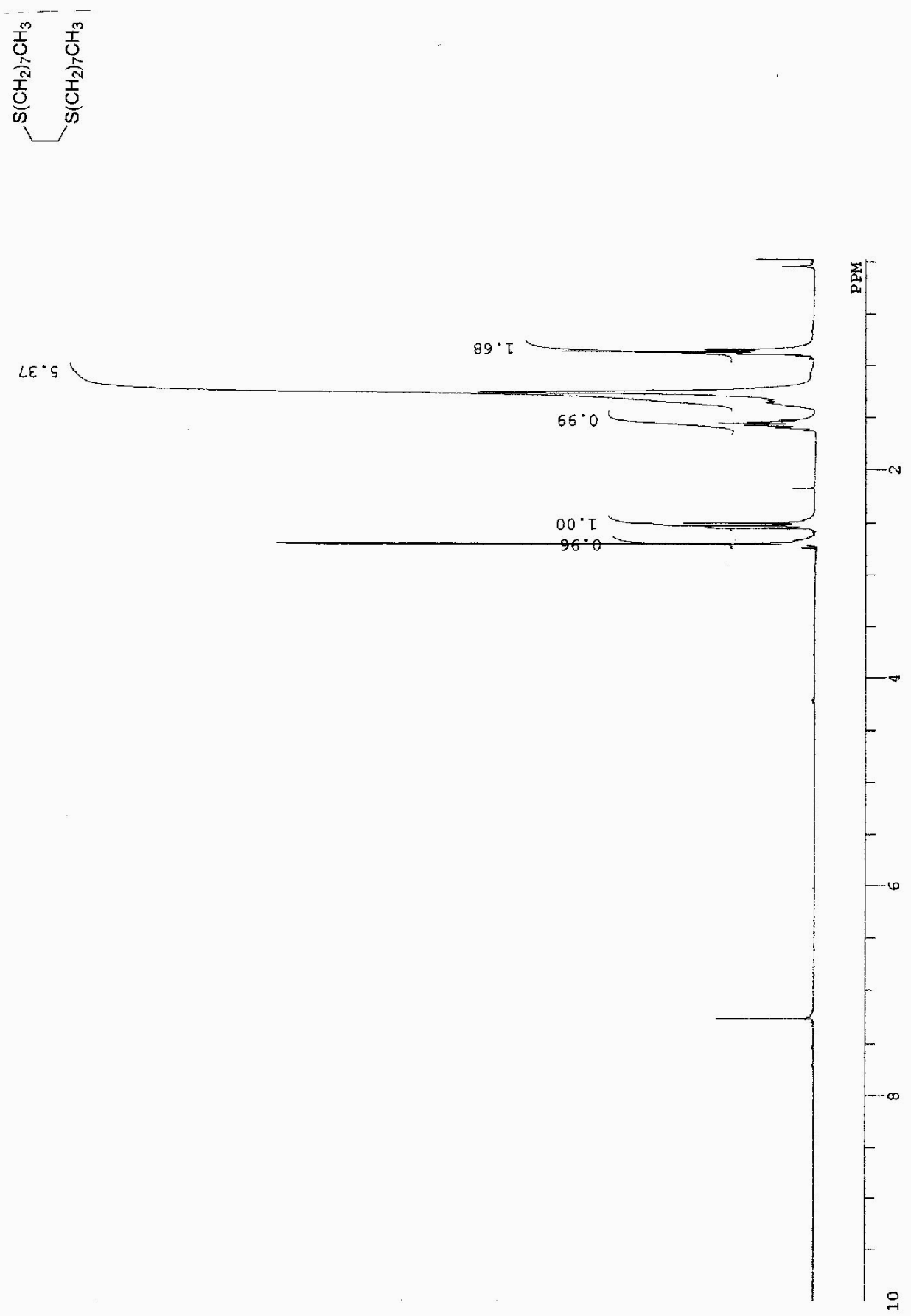

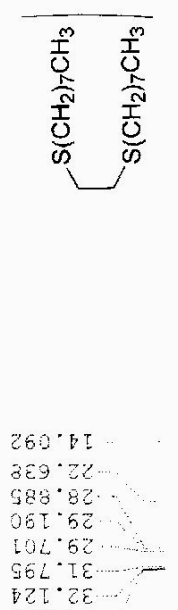

$96 L^{\circ}$ TE

कEI $2 \varepsilon$

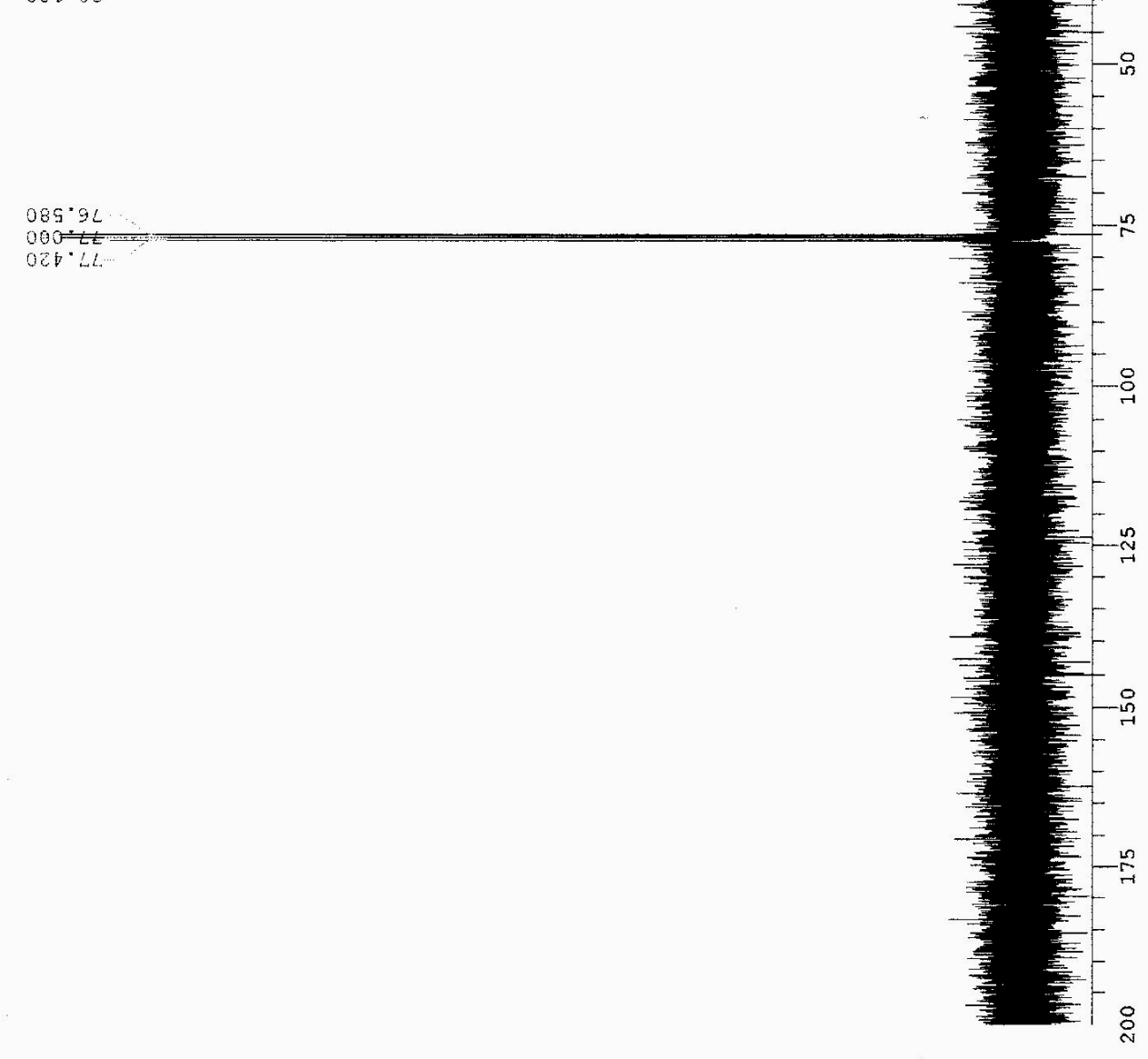




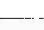

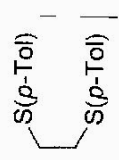

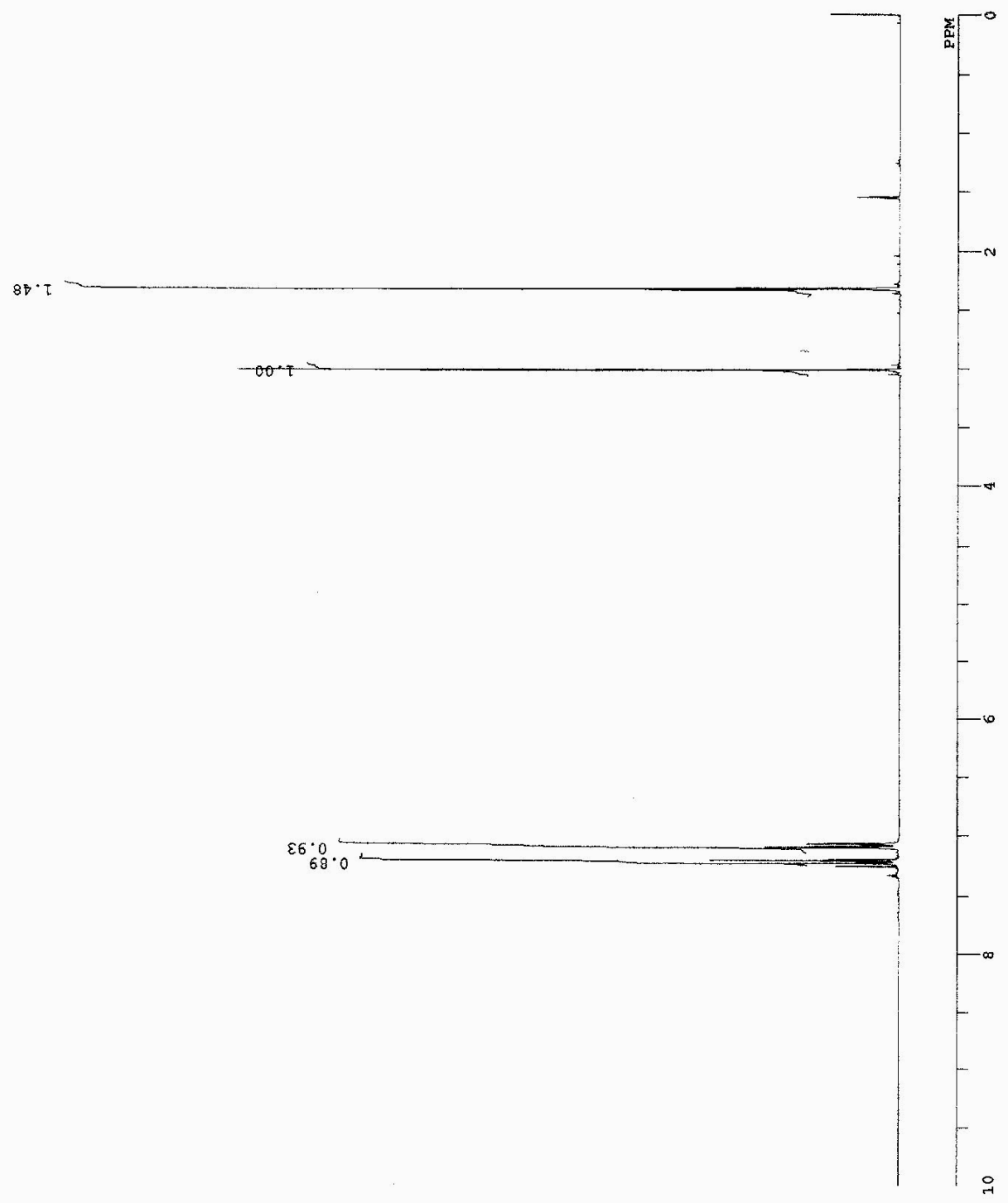




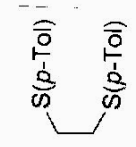

$900^{\circ} \mathrm{IZ}$

$\$ 66^{\circ} 89$

tL9. 9

0002

$027^{\circ}$ L

E9L.62T

GLU LEL

GI $9 \varepsilon \mathrm{T}$

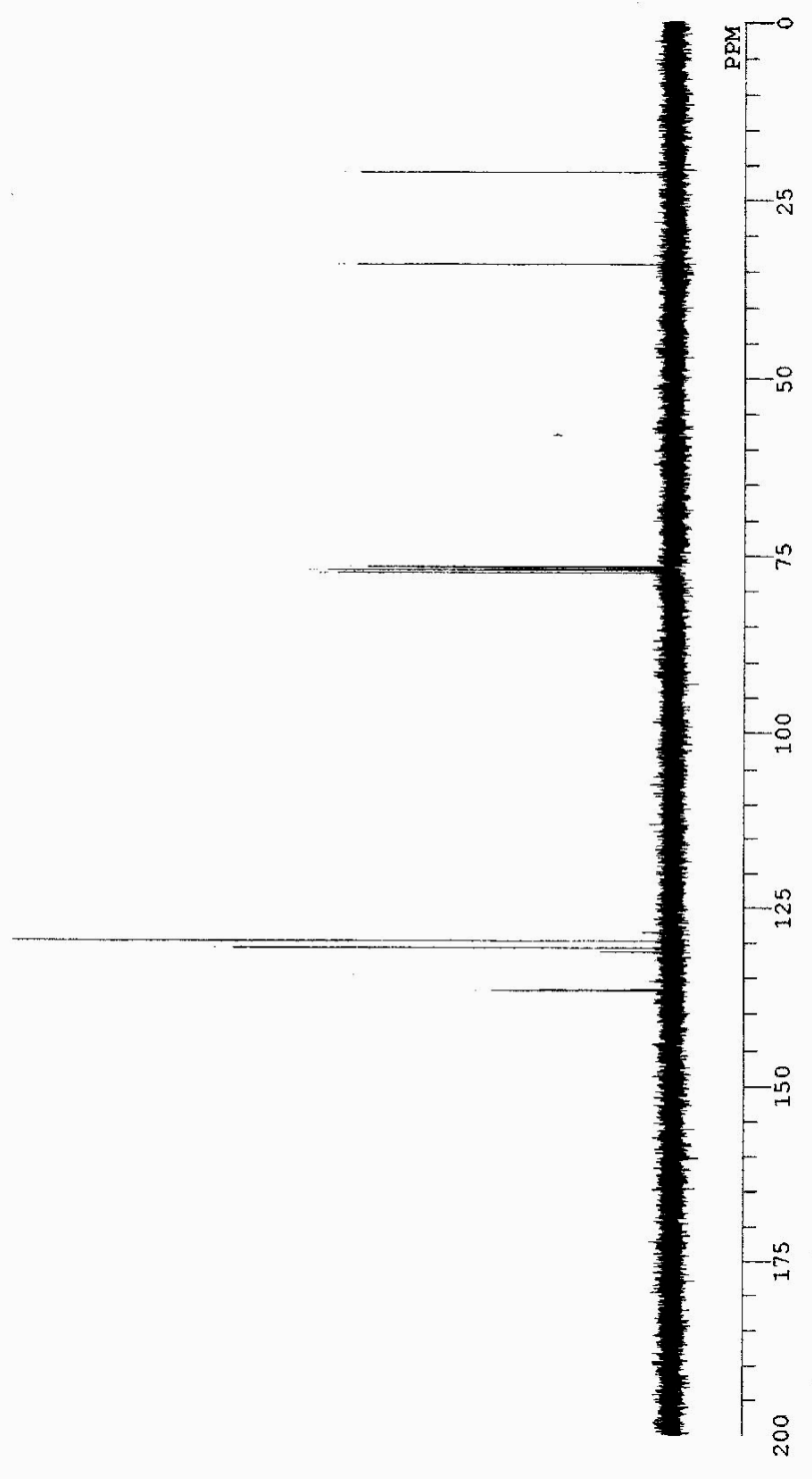

S-23 
$0=\underbrace{\frac{1}{0}}_{\substack{I \\ \frac{1}{N}}}$

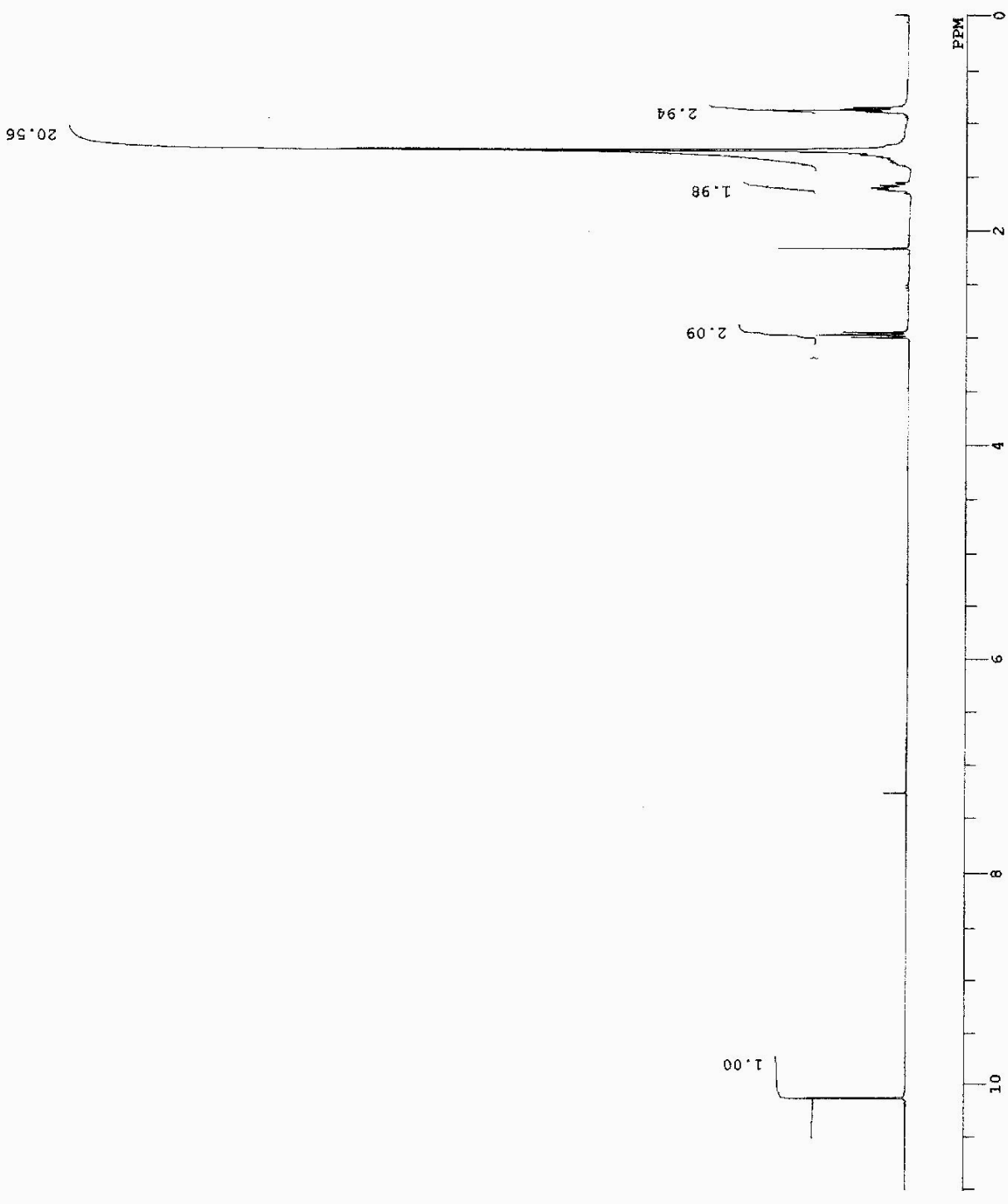



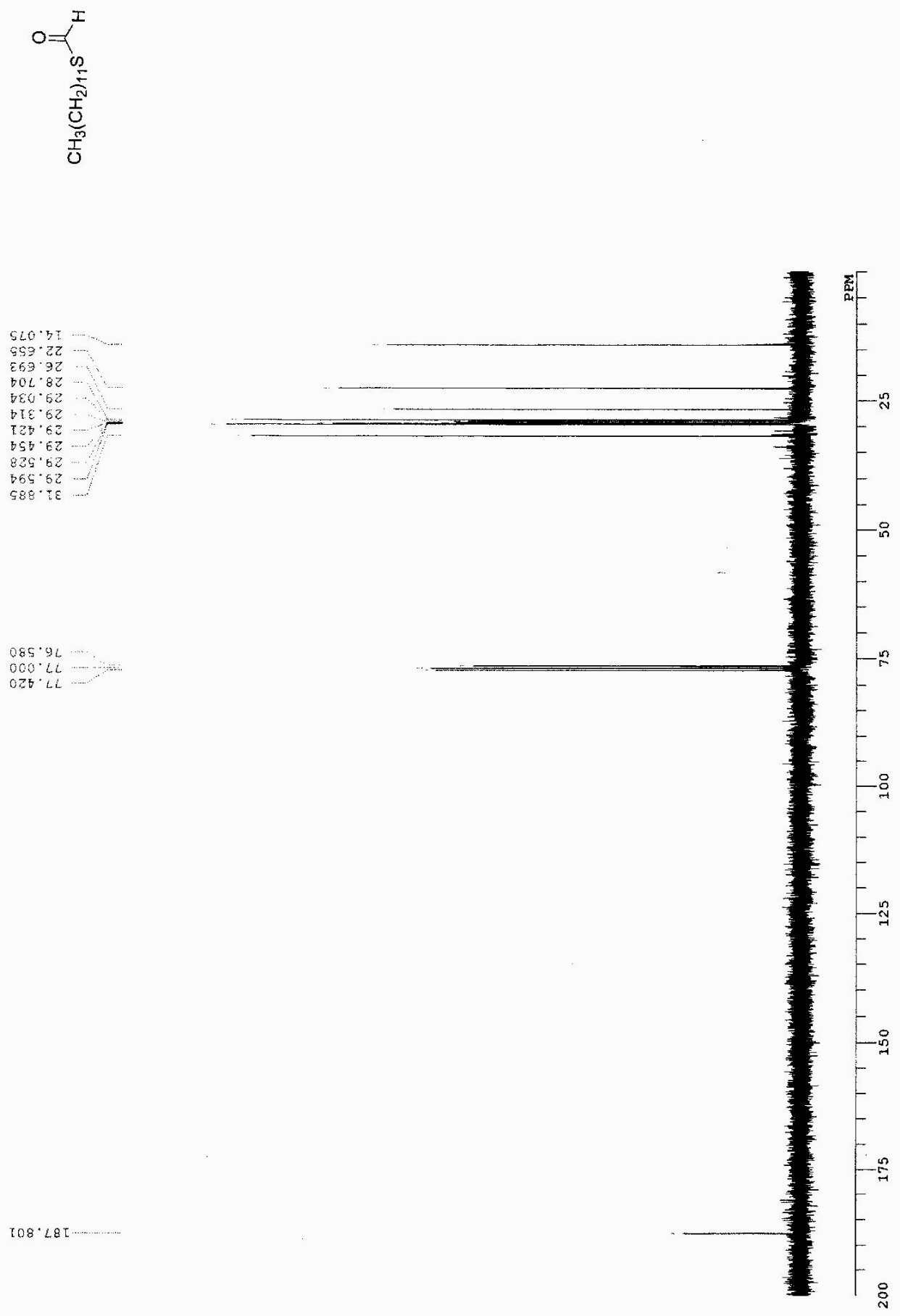

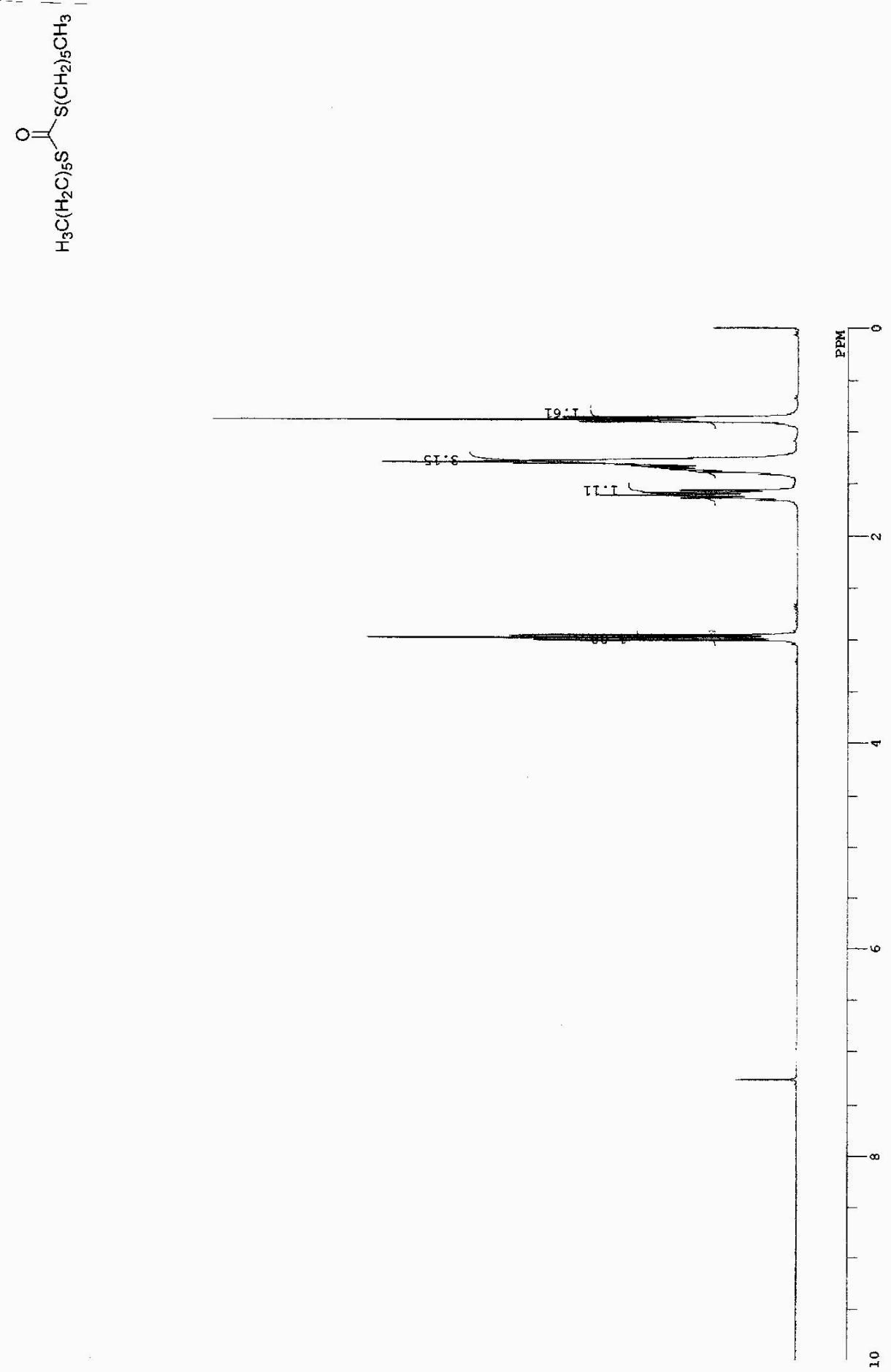

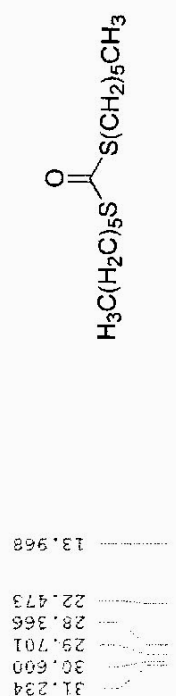

$085^{\circ} 9$

$627.2 L$

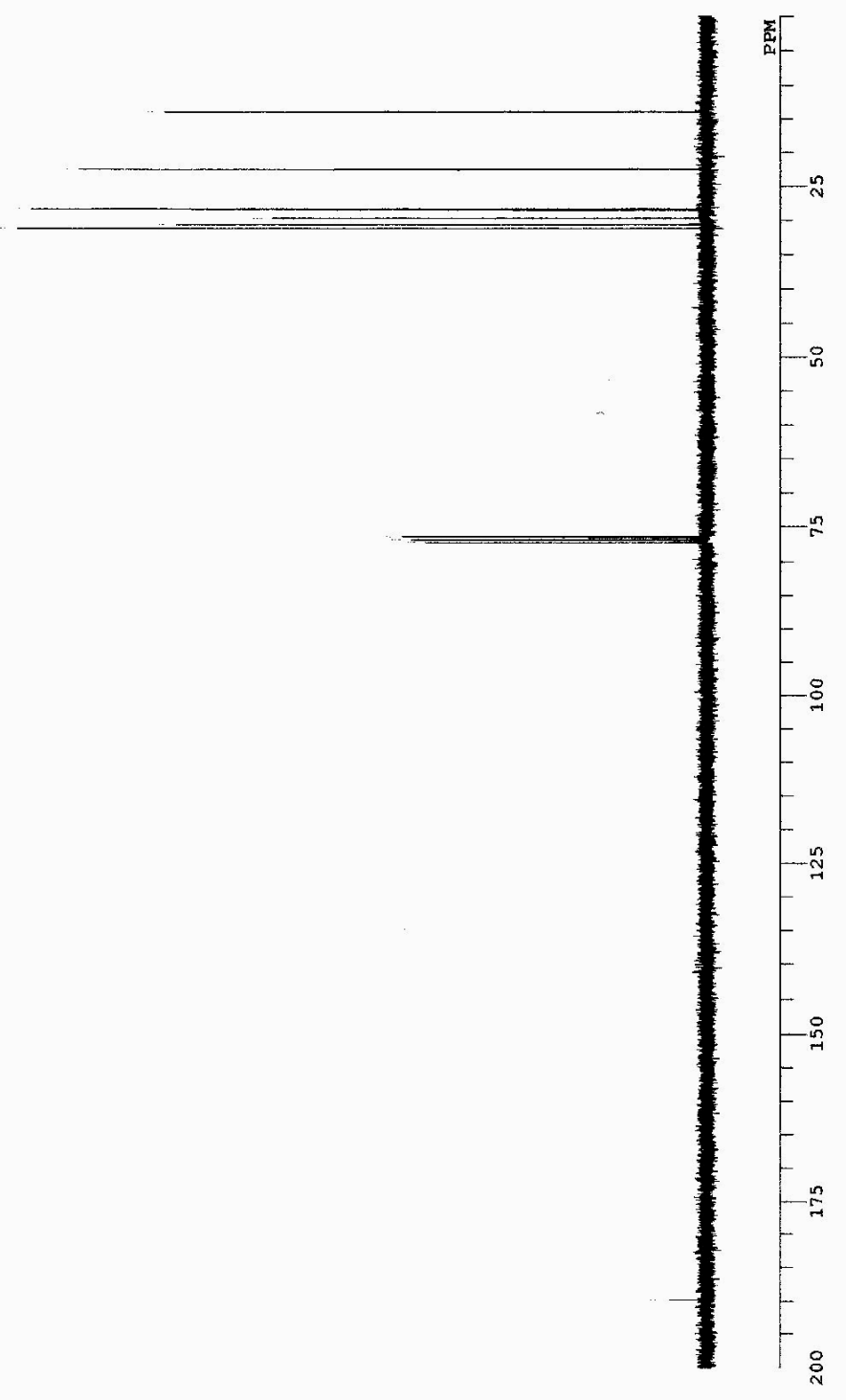




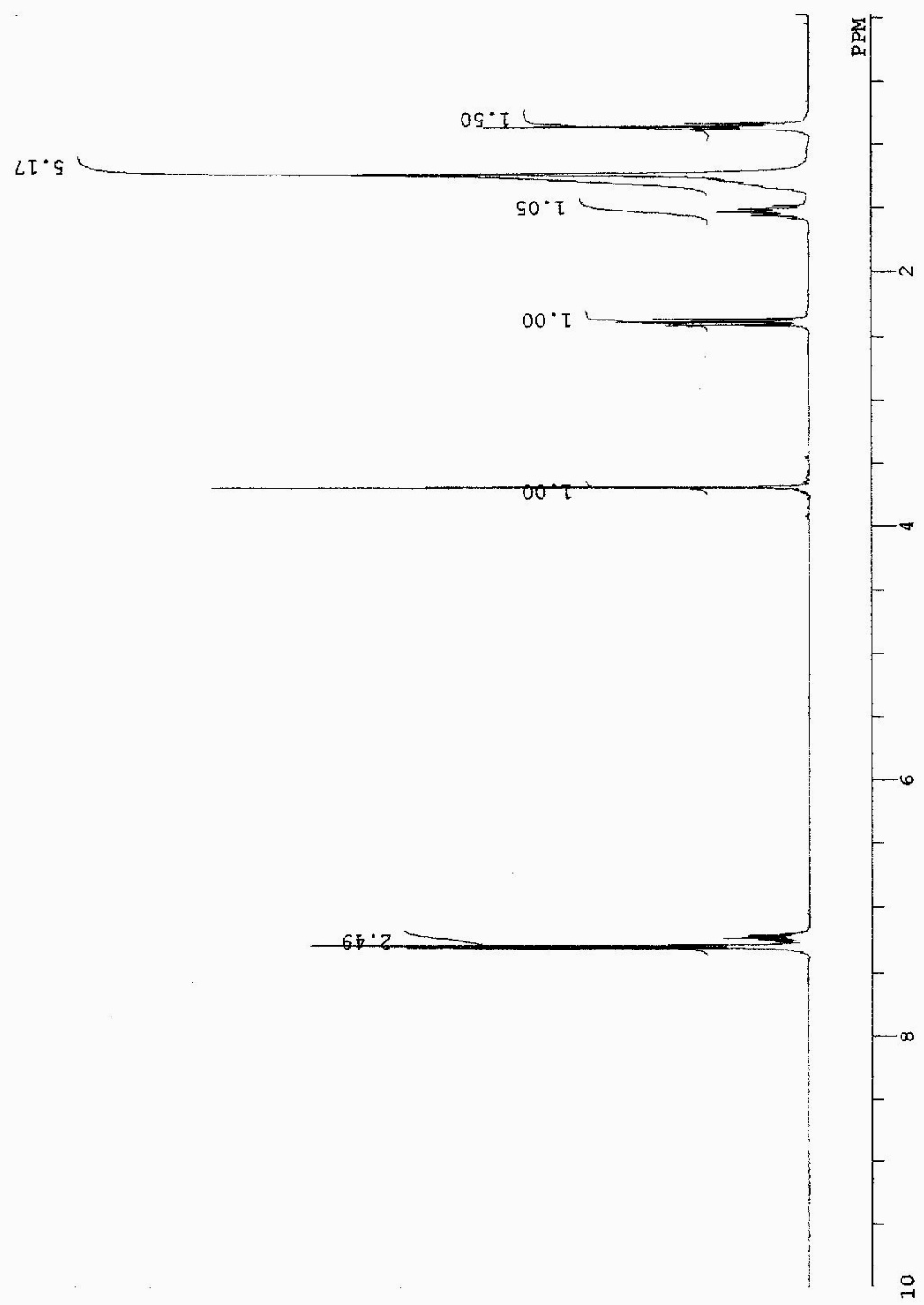




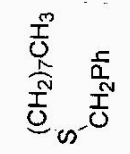

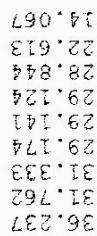

09592

$000^{\circ} L L$
$0 Z 0^{\circ} \angle L$

$88 \mathrm{i} 92 \mathrm{I}$

$8 L E \cdot 8 Z T$
$99 L \cdot 8 Z T$

$6 \varepsilon 9 \cdot 8 \varepsilon t$

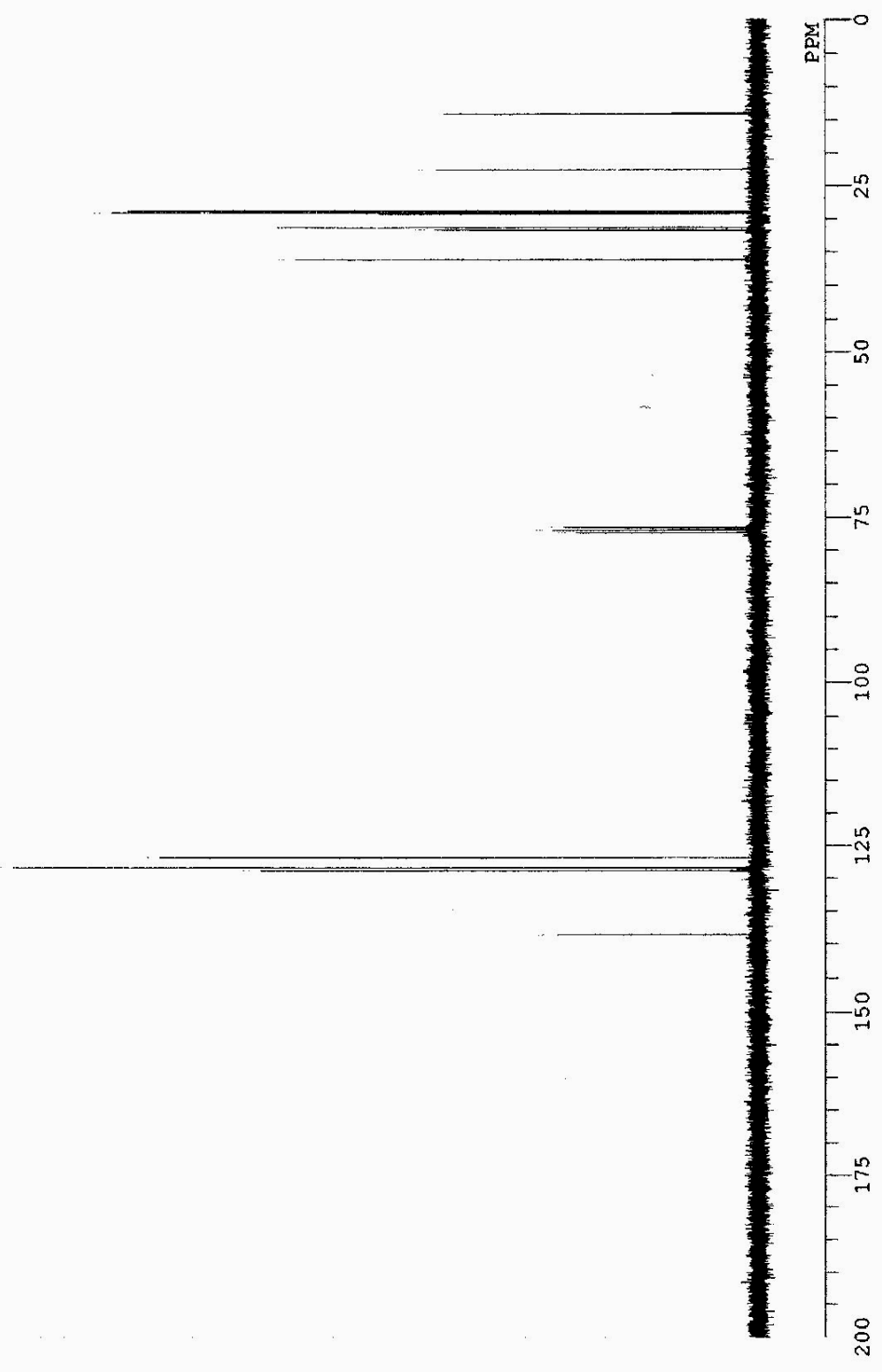




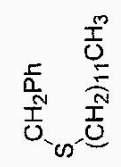

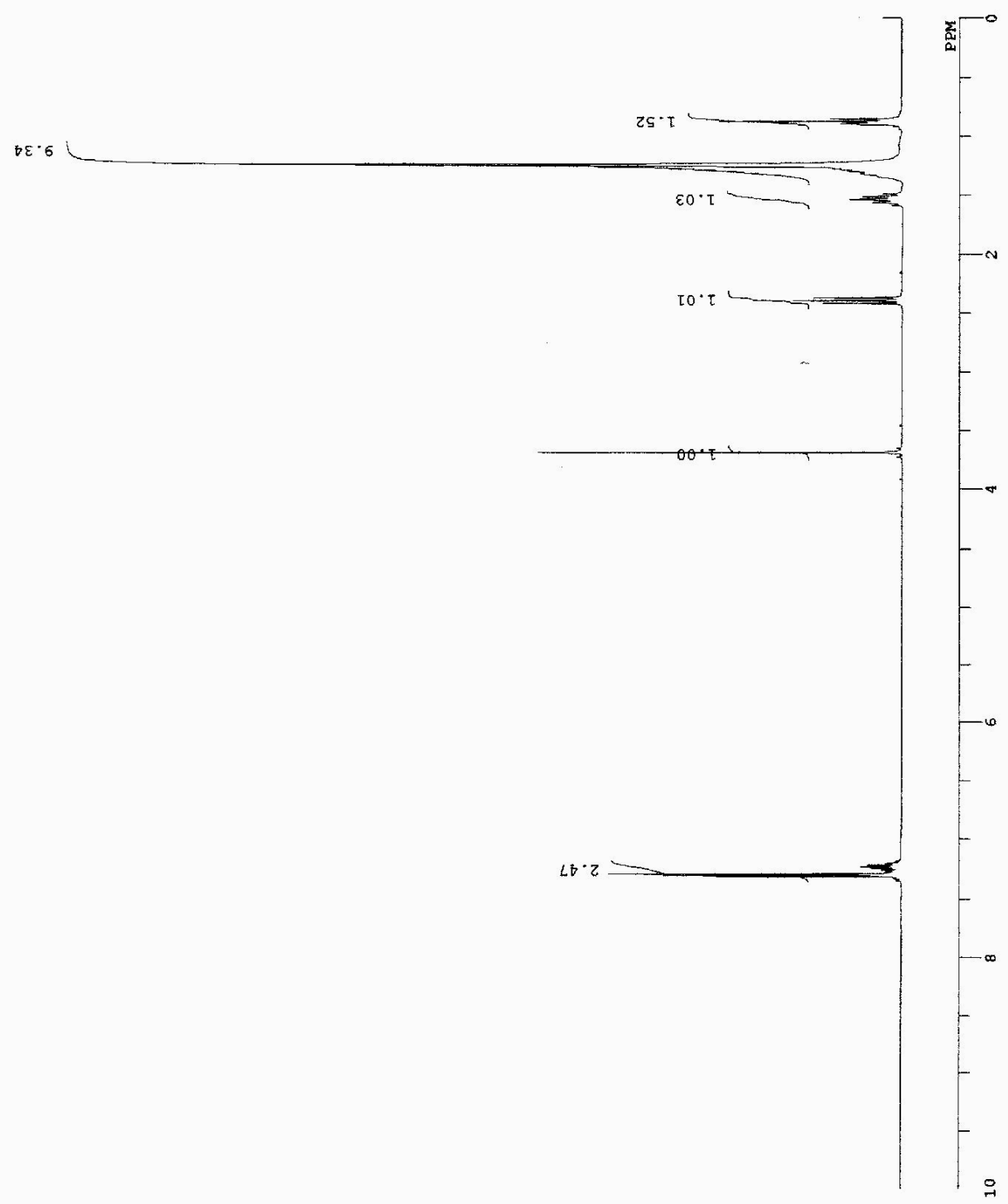




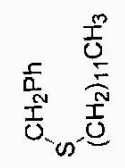

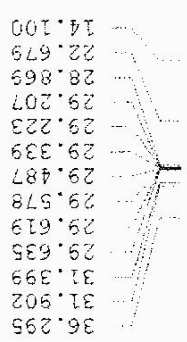

$089 \cdot 94$

$000^{\circ}-2 L$

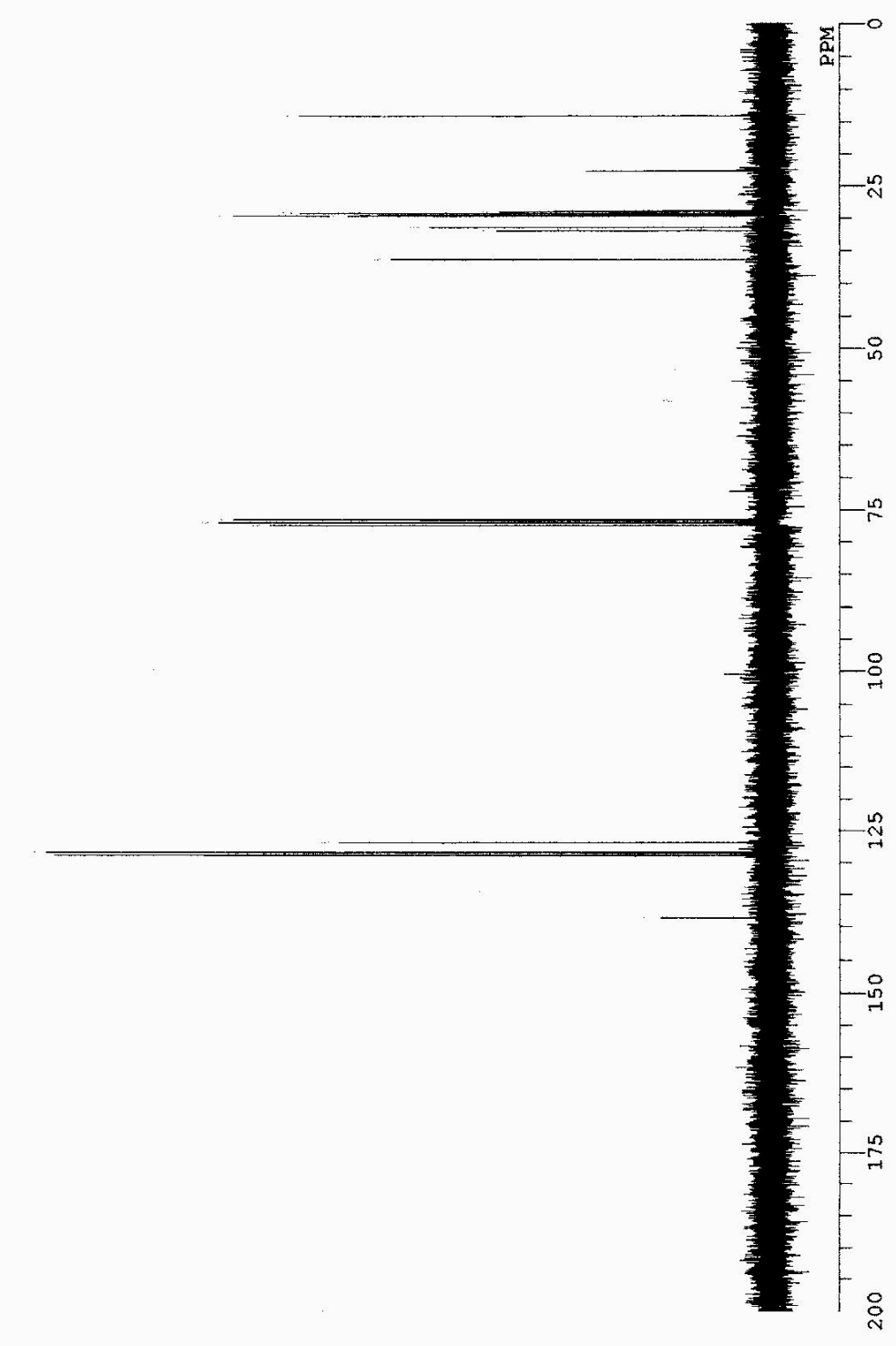

$528 \cdot 92 \pm$
$5.82 \mathrm{I}$

$408^{\circ} 8 \mathrm{I}$

$039^{\circ} \mathrm{BEI}$ 


$$
0=
$$

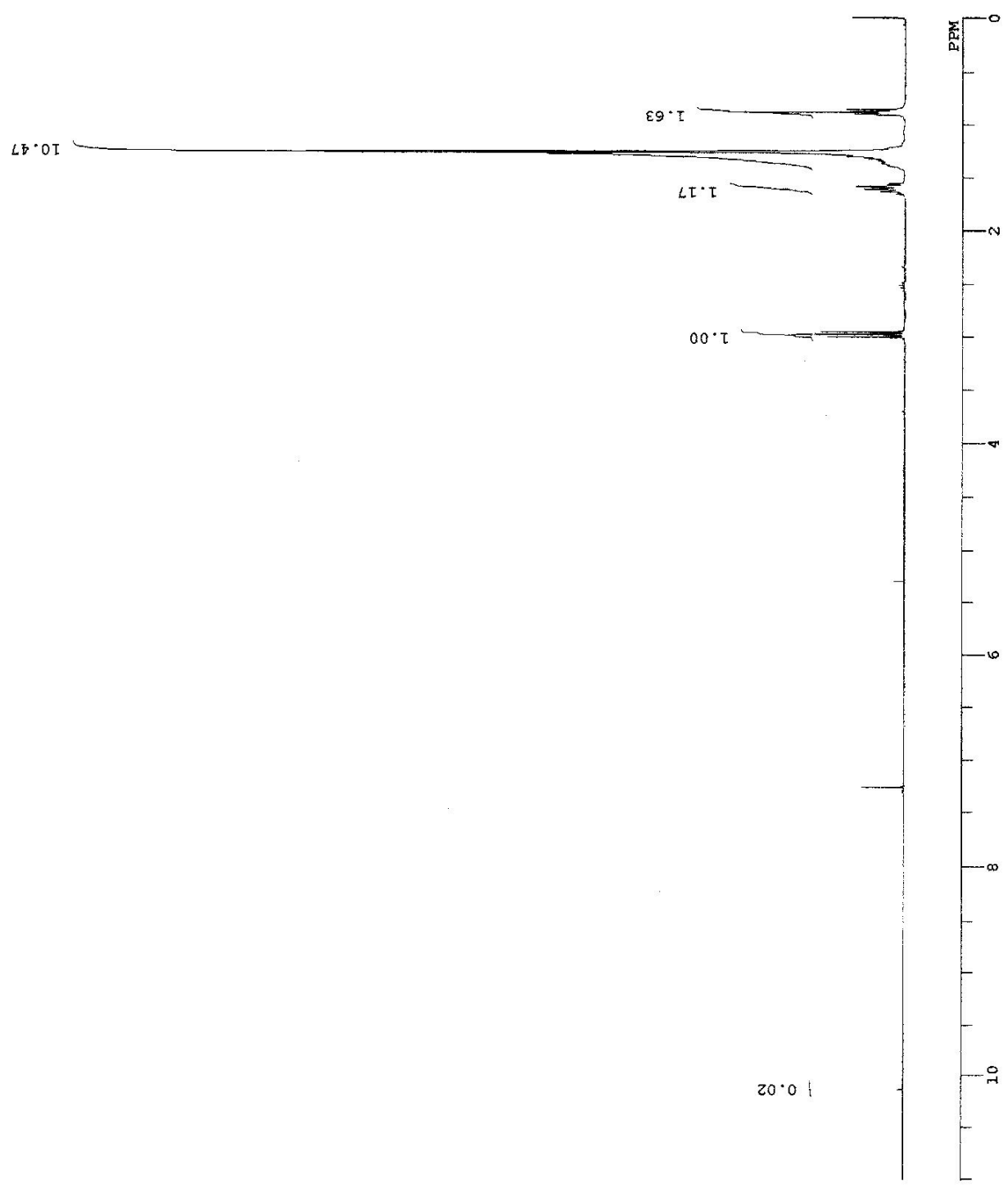



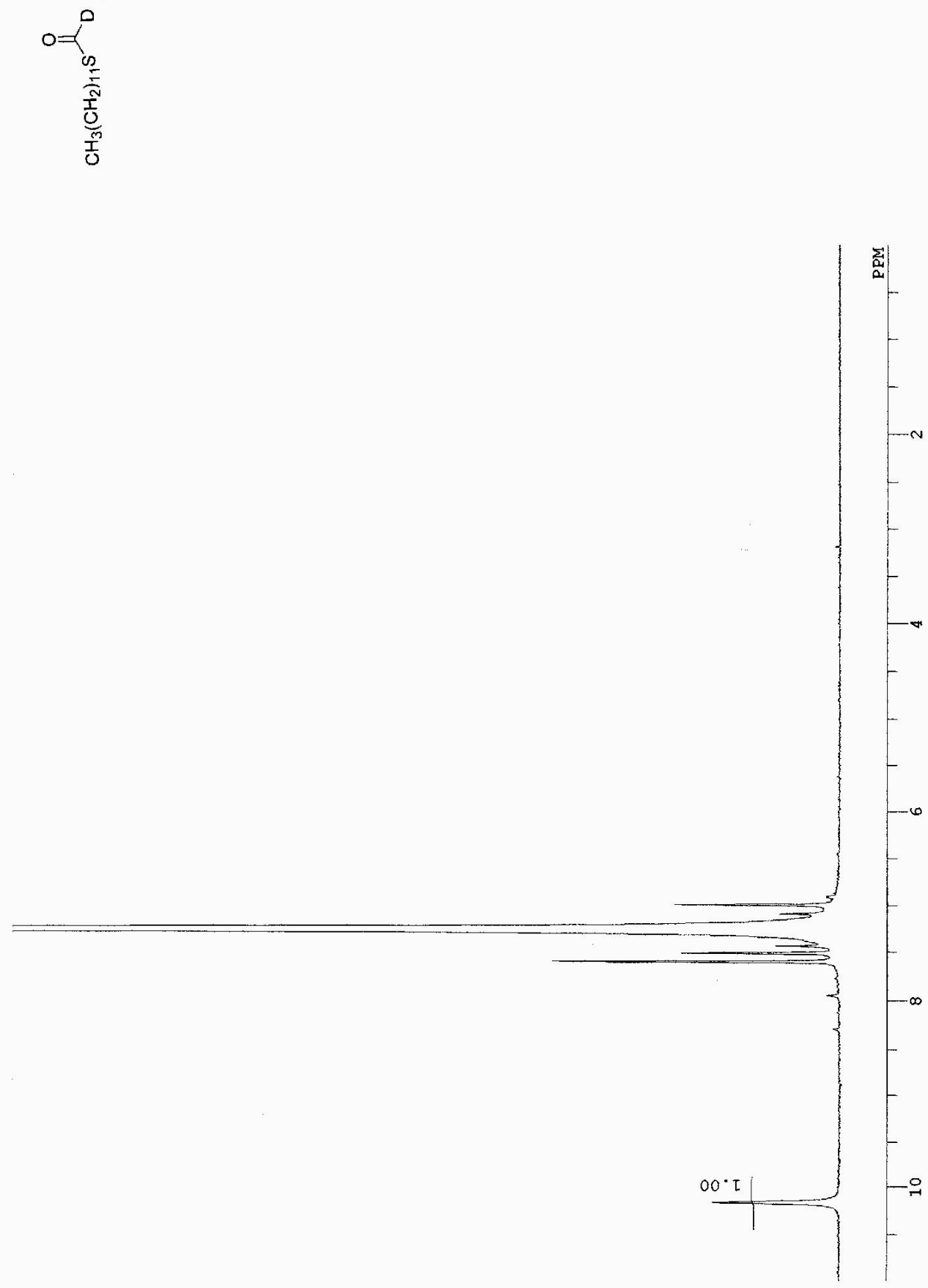

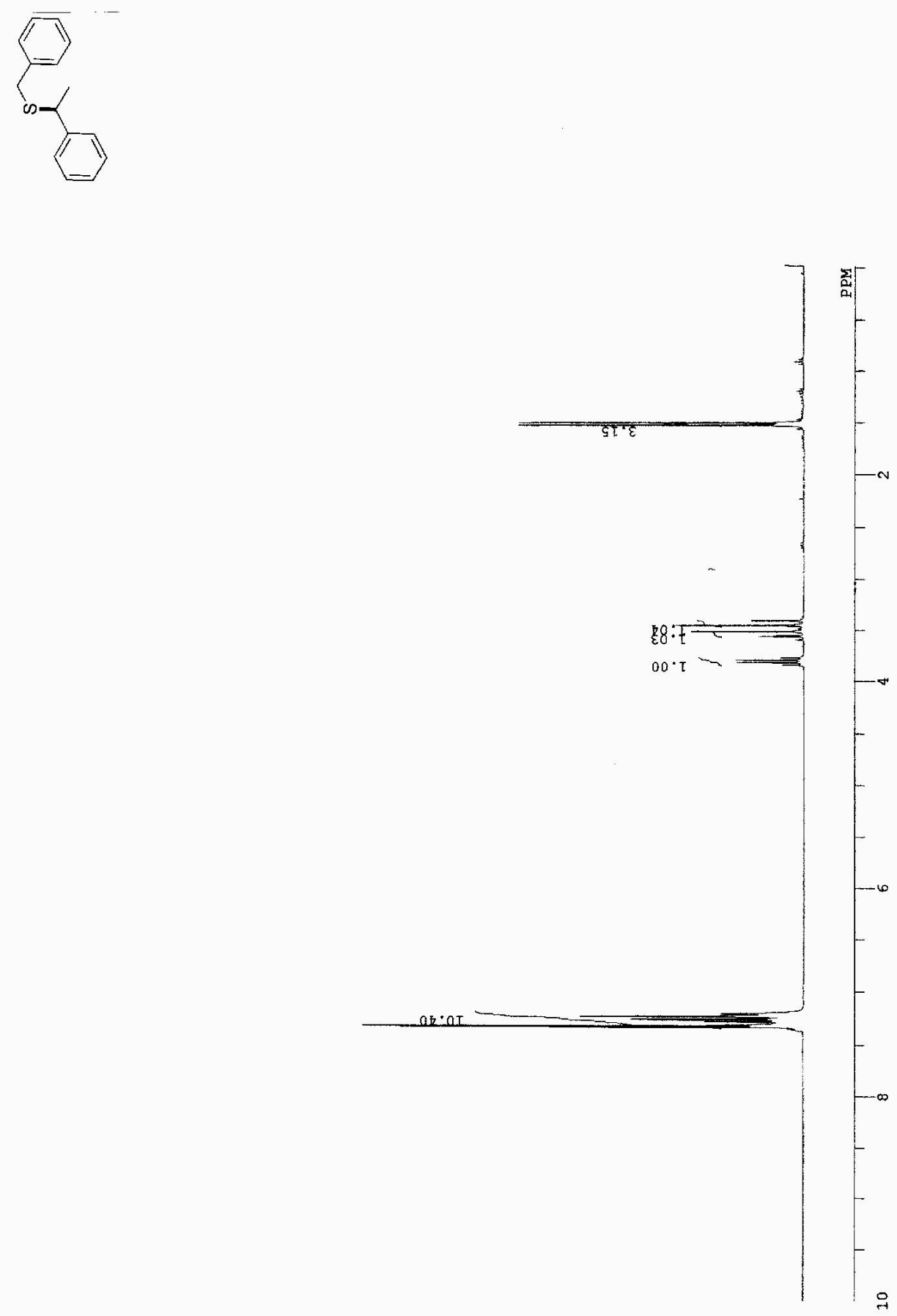

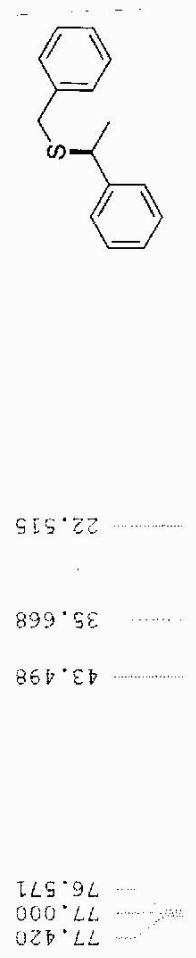

$96 L \cdot 9 Z t$

जट०. LEI

मGE.

19. 8

858.82

$\overrightarrow{<} 6 \varepsilon \cdot 8 \varepsilon t$

दิเ है

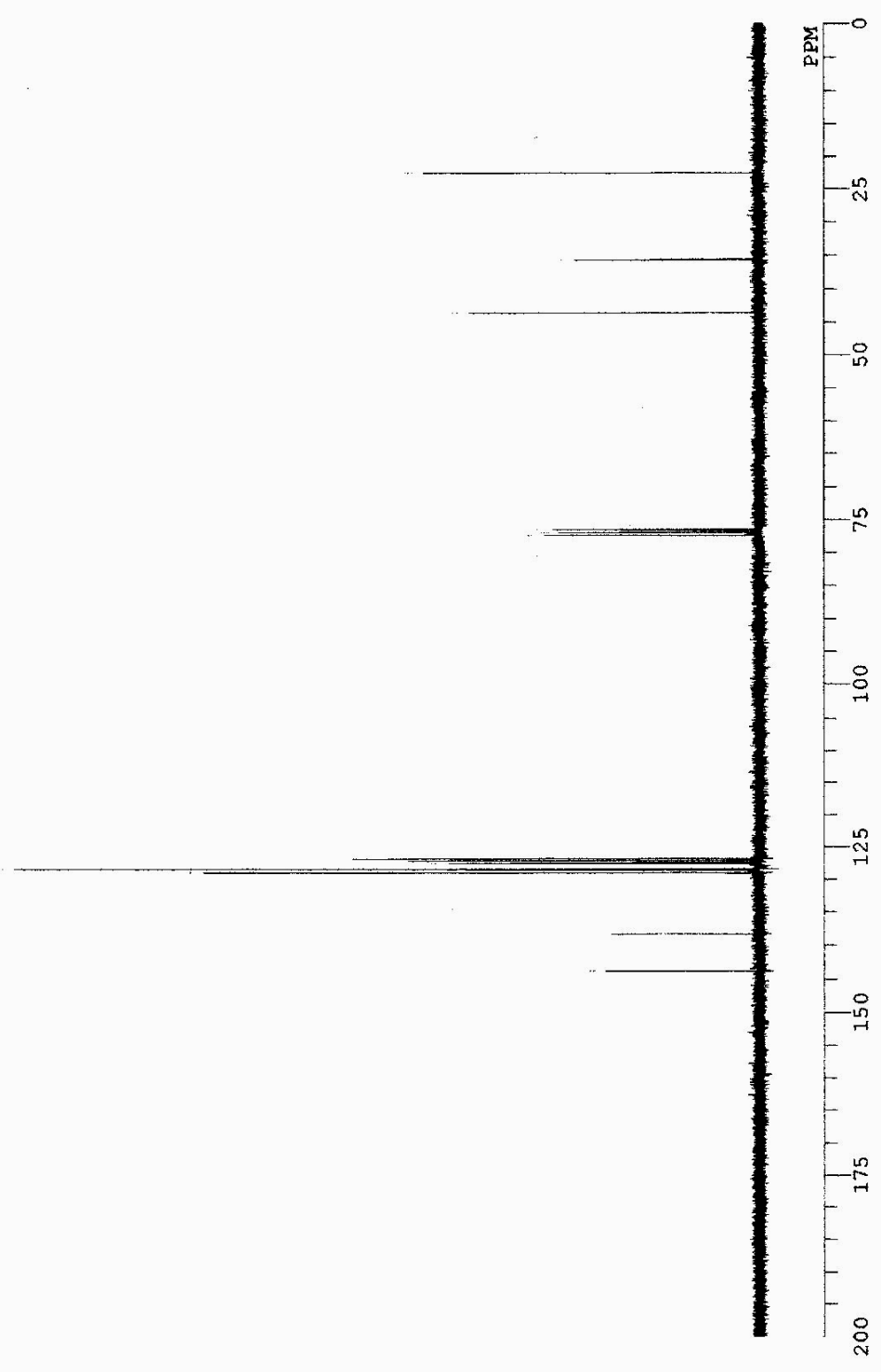

